\title{
Multiplets of free d- and f-metal ions: A systematic DFT study
}

\author{
Alain Bore ${ }^{(1)}$ and Claude A. Daul ${ }^{(2)}$
}

(1) Laboratoire de Chimie Inorganique et Bioinorganique, Ecole Polytechnique Fédérale de Lausanne, CH-1015 Lausanne, Switzerland

(2) Département de Chimie, Université de Fribourg, Chemin du Musée 9, CH-1700 Fribourg, Switzerland

\begin{abstract}
In this work we apply in a systematic way our multi-determinantal model to calculate the fine structure of the whole atomic multiplet manifold. The key feature of this approach is the explicit treatment of near degeneracy correlation using ad hoc Configuration Interaction (CI) within the active space of Kohn-Sham (KS) orbitals with open d- or f-shells. The calculation of the CImatrices is based on a central symmetry decomposition of the energies of all single determinants (micro-states) calculated according to Density Functional Theory (DFT) for frozen KS-orbitals corresponding to the averaged configuration, eventually with fractional occupations, of the d- or forbitals and/or the direct calculation of the electrostatic reduced matrix elements (Racah or SlaterCondon parameters) occurring in the corresponding active space. We performed DFT calculations on all divalent and trivalent $\mathrm{d}^{2}-\mathrm{d}^{8}$ metal ions, as well as the $\mathrm{f}^{2}-\mathrm{f}^{12}$ lanthanide(III) ions. We compare the results of both variants of the method with the data available in the literature. Both procedures yield multiplet energies with an accuracy of about hundred wave numbers and fine structure splitting accurate to less than a tenth of this amount.
\end{abstract}

\section{Keywords:}

Density Functional Theory, Kohn-Sham equation, electrostatic repulsion, Racah parameters, Slater Condon parameters, Self Interaction Correction. 


\section{Introduction}

LFDFT, a DFT-based ligand field theory, has been introduced recently as a tool for the parameterfree calculation of optical and magnetic properties of transition metal complexes ${ }^{[1]}$ This method, based on the multiplet theory of transition metal ions, uses a series of DFT calculations to calculate the ligand field, interelectron repulsion and spin-orbit coupling parameters acting on the frontier orbitals of the molecule. One can then predict the ground and excited states of a complex within the framework of ligand field theory. Originally developed for $d$ metal complexes, ${ }^{[1-3]}$ the method can be generalized to other systems, such as $f$ elements compounds. This was demonstrated using examples such as rare earth hexach loro complexes ${ }^{[4]}$ and the gadolinium(III) aqua ion. ${ }^{[5]}$ Of course, the accuracy of the method is directly related to the accuracy of DFT in the determination of the Hamiltonian parameters. Thus, a quantitative prediction of the excited state energies depends on an accurate description of all interactions. In this work, we present a detailed study of the electron repulsion parameters.

The one-electron theory of atoms, molecules, and crystals has enjoyed a wide success in many branches of physics and chemistry. This theory provides a physically appealing description of the electronic structure of many-electron systems. In addition to its formal and conceptual aspects, this theory provides a convenient basis for performing detailed calculations for specific many-electron systems. In such calculations, it is usually necessary to introduce many simplifying assumptions in order to make progress.

The one-electron wave functions for a many-electron system can be determined by the variational method. One begins by constructing an approximate many-electron wave function $\psi$ in terms of a set of one-electron wave functions or spin-orbitals $\Phi(\beta)$. Using this representation for $\psi$, the average energy of the system is determined with respect to the $\Phi(\beta)$. This average energy is then varied with respect to the $\Phi(\beta)$. This leads to a set of variational equations for the $\Phi(\beta)$. From the standpoint of the variational principle, the solutions of these equations are the best spin-orbitals consistent with the representation originally chosen for $\psi$. The variational equations have the form of coupled integro-differential equations. In principle, a self-consistent set of solutions can be obtained by iterative techniques.

The actual form of the variational equations, or the self-consistent field equations, as they are more commonly called, is dependent on the representation chosen for $\psi$. If the expression for $\psi$ in terms of the $\Phi(\beta)$ is very restrictive, or if the $\Phi(\beta)$ themselves are subject to restrictions, the accuracy of the overall solution will be adversely affected. On the other hand, it may be necessary to impose such restrictions in order to simplify an otherwise in tractable problem.

In its simplest form, the Hartree-Fock approximation treats only a single electronic configuration. In 
this case, $\psi$ is represented by a linear combination of determinantal wave functions. Each of these corresponds to a different assignment of the electronic quantum numbers of the constituent spinorbitals consistent with the symmetry of the chosen configuration. In the special case of a closed shell configuration, there is only one possible assignment of these quantum numbers, and hence $\psi$ is here represented by a single determinantal wave function.

In the configuration interaction representation, which is an extension of the Hartree-Fock method, $\psi$ is expanded in terms of the determinantal wave functions associated with more than one configuration. The ordinary Hartree-Fock method is normally used when the configuration of interest is far removed (in total energy) from all other configurations, or when the system is so complicated that a superposition of configurations is prohibitively difficult. Configuration interaction is most commonly taken into account when two or more configurations are expected to interact strongly because their total energies are nearly equal. Since the amount of effort required to obtain a solution for even a single configuration is quite formidable, a superposition of configurations is usually employed only when this is absolutely essential, and then only in the case of the simplest systems.

In the Hartree-Fock model, the coulomb correlations are again ignored, but a correlation in the motion of pairs of electrons of like spin is introduced through the use of determinantal wave functions. This is called statistical correlation because it is associated with the fact that electrons obey the Fermi-Dirac statistics. The effect of the statistical correlation is to keep electrons of like spin away from each other, in keeping with the requirements of the Pauli exclusion principle. Since the coulomb correlation between a pair of electrons is most important when these electrons are close together, and since electrons of like spin are kept apart by the statistical correlation, the neglect of coulomb correlation between like spin electrons in the Hartree-Fock model is offset in large measure by the inclusion of statistical correlation.

In the configuration interaction representation, the coulomb correlation between electrons of unlike spin can be taken into account, as can the residual spatial correlation between like spin electrons. (This residual correlation is the difference between the instantaneous coulomb correlation and the statistical correlation.) Unfortunately, the treatment of correlation effects by the method of configuration interaction does not appear to be a practical one for any but the simplest manyelectron systems.

\section{Central Field Approximation}

We shall now focus our attention on free atoms and ions, our main interest in the present communication. The self-consistent field equations for an atomic system can be simplified considerably by making use of the central field approximation. In this approximation, the spin- 
orbitals are expressed as products of radial wave functions $\mathrm{R}(\mathrm{r})$, angular wave functions or spherical harmonics $\mathrm{Y}(\theta, \Phi)$, and spin functions $\mu$. The spin-orbitals for a central field are characterized by the four quantum numbers $n, \lambda, m$, and $s$, where $n$ is the principal quantum number, $\lambda$ the azimuthal or orbital quantum number, $\mathrm{m}$ the magnetic quantum number, and $\mathrm{s}$ the spin quantum number. (For typographical convenience, the symbol $\lambda$ will be used in place of the more conventional script "el.") The most general central field representation for $\Phi(\beta)$ is

$$
\phi(\beta)=\phi(n, \lambda, m, s)=R_{n \lambda m s}(r) Y_{\lambda m}(\theta, \phi) \mu_{s}
$$

a somewhat more restrictive representation is

$$
\phi(\beta)=\phi(n, \lambda, m, s)=R_{n \lambda s}(r) Y_{\lambda m}(\theta, \phi) \mu_{s}
$$

a still more restrictive representation is

$$
\phi(\beta)=\phi(n, \lambda, m, s)=R_{n \lambda}(r) Y_{\lambda m}(\theta, \phi) \mu_{s}
$$

In (1), there may be a different radial wave function for each set of four quantum numbers; in (2), the radial wave function is independent of $\mathrm{m}$; and in (3), the radial wave function is independent of both $\mathrm{m}$ and $\mathrm{s}$.

Applying the method of separation of variables, the three-dimensional spatial wave equations are reduced to one-dimensional (radial) wave equations, which can be solved by standard techniques. There will be as many different radial wave equations as there are different radial wave functions. Representation (3) is the one most commonly used, since this leads to the fewest radial wave equations. In some applications it may be desirable to use the more flexible representations (1) or (2), but this involves considerably greater effort.

The amount of effort required to solve the Hartree-Fock equations based on the representation (3) goes up rapidly as the number of occupied orbitals $(\mathrm{n} \lambda)$ increases. Not only is it necessary to solve a different radial wave equation for each orbital, it is also necessary to calculate a different exchange potential for each orbital. Since each of these exchange potentials is determined by the sum of exchange integrals whose number increases rapidly as the number of occupied orbitals increases, the calculation of the exchange terms becomes more and more overwhelming as the number of orbitals becomes large. The computational difficulties would be increased even further if the representations (1) or (2) were employed in place of (3).

\section{Density functional theory}

Density functional theory (DFT) is one of the most popular approaches to quantum mechanical 
many-body electronic structure calculations of molecular and condensed matter systems. For the sake of completeness let us give here a short description of the theory.

Traditional methods in electronic structure, like Hartree-Fock theory are based on the complicated many-electron wavefunction. The main objective of density functional theory is to replace the many-body electronic wavefunction with the electronic density as the basic quantity. Whereas the many-body wavefunction is dependent on $3 N$ variables, three spatial variables for each of the $N$ electrons, the density is only a function of three variables and is a simpler quantity to deal with both conceptually and practically.

Although density functional theory has its conceptual roots in the Thomas-Fermi model, DFT was not put on a firm theoretical footing until the Hohenberg-Kohn $(\mathrm{HK})$ theorem ${ }^{[6]}$ which demonstrates the existence of a one-to-one mapping between the ground state electron density and the ground state wavefunction of a many-particle system. Moreover, the HK theorem proves that the ground state density minimizes the total electronic energy of the system. Since the HK theorem holds only for the ground state, DFT is also a ground state theorem.

The Hohenberg-Kohn theorem is only an existence theorem, stating that the mapping exists, but does not provide any such exact mapping. It is in these mappings that approximations are made. The most popular such mapping is local-density approximation (LDA), which gives an approximate mapping from the density of the system to the total energy. The LDA is exact for the uniform electron gas, also known as jellium.

In practice, the HK theorem is not often used to directly make calculations. Instead, the most common present-day implementation of density functional theory is through the Kohn-Sham method. ${ }^{[7]}$ Within the framework of Kohn-Sham DFT, the intractable many-body problem of interacting electrons in a static external potential is reduced to a tractable problem of noninteracting electrons moving in an effective potential. The effective potential includes the external potential and the effects of the Coulomb interactions between the electrons.

In many cases DFT with the local-density approximation gives quite satisfactory results in comparison to experimental data at relatively low computational costs when compared to other ways of solving the quantum mechanical many-body problem.

DFT has been very popular for calculations in solid-state physics since the 1970s. However, it was not considered accurate enough for calculations in quantum chemistry until the 1990s, when the approximations used in the theory were greatly refined. DFT is now a leading method for electronic structure calculations in both fields.

\section{Early Models: Free-Electron Exchange Approximation}

Since the principal computational difficulty posed by the Hartree-Fock equations is the treatment of the exchange terms, it would be very desirable to simplify the treatment of these terms. It was 
pointed out by Slater that the Hartree-Fock exchange potentials for different occupied orbitals, though different from one another in detail, are similar in many respects. This suggested to Slater that the essential features of the Hartree-Fock method could be retained by replacing the exchange potentials for different orbitals by a universal exchange potential formed by suitably averaging these individual exchange potentials. ${ }^{[8]}$

This approximation in itself does not overcome the difficulty of calculating exchange potentials from exchange integrals, since it is still necessary to obtain all the individual exchange potentials prior to taking their average. However, the physical nature of the averaged exchange potential can be interpreted in fairly concrete terms, and this interpretation suggests a method of approximation which is strikingly simple, and which avoids the need to calculate exchange integrals altogether. Let us consider, then, the physical interpretation of the averaged exchange potential.

In electrostatics, the coulomb potential can be expressed in terms of the (actual) charge density. By analogy, the exchange potential can be expressed in terms of a fictitious charge density known as the exchange charge density. More precisely, the exchange potential acting on an electron in a given orbital can be considered to arise from an exchange charge density whose detailed form depends on the particular orbital. Although the exchange charge density is different in detail for different orbitals, it has certain features, which are common to all (occupied) orbitals. Since the exchange potential for a given orbital is determined by the corresponding exchange charge density, and since the exchange charge densities for different orbitals are similar in many respects, the exchange potentials for different orbitals may also be expected to be similar in many respects. In view of these similarities, it is meaningful to construct an averaged exchange potential, and it is reasonable to expect this averaged exchange potential to represent each of the individual exchange potentials fairly well.

Just as the exchange potential can be averaged over all orbitals, so can the exchange charge density. In this connection, it is instructive to consider a free-electron gas, for which the averaged exchange potential and the averaged exchange charge density can be determined exactly.

In a free-electron gas, the averaged exchange charge density (i) has a to tal content equal to plus one electronic charge; and (ii) has a value at the position of the electron on which it acts equal in magnitude to the total charge density associated with all the electrons having the same spin as this electron. Moreover, the averaged exchange density follows the motion of the electron on which it acts, and is always localized in the neighbourhood of this electron. In particular, the averaged exchange charge density is spherically symmetrical, and is centred at the position of this electron, where it attains its maximum value, which is given by (ii) above.

In physical terms, the exchange charge represents the removal of one electronic charge from the set of electrons having the same spin as the electron on which the corresponding exchange potential 
acts. This deficiency of one electronic charge is also known as the exchange or Fermi hole. The exchange hole arises from the operation of the Pauli exclusion principle, and describes the tendency of electrons of like spin to avoid one another.

In a free-electron gas, the averaged exchange potential is proportional to the cube root of the electronic charge density. Since the latter quantity is uniform in space, the averaged exchange potential is a spatially constant quantity whose magnitude depends only on the electronic charge density. Denoting the total electronic charge density (both spins) by $\rho$, and assuming equal numbers of up and down spins, the free-electron exchange potential is given by

$$
V_{X}[\rho]=-6\left[\frac{3}{8 \pi} \rho\right]^{1 / 3}
$$

where, as elsewhere in this section, distances are measured in Bohr units and energies in Rydberg units.

The exchange hole in an atomic system has some but not all the attributes of the exchange hole in a free-electron gas. In both cases, the exchange hole has a total content of plus one electron ic charge, and a value at the position of the electron equal in magnitude to the total like-spin electronic charge density, i.e., $[\rho / 2]$. To the extent that the exchange hole in an atomic system depends on the local like-spin electronic charge density, the averaged exchange potential for such a system may also be expected to depend on this quantity, by analogy with the free-electron case. If we adopt the extreme view, as Slater did, and assume that the averaged exchange potential in a non-uniform system such as a free atom depends only on the local electronic charge density, we are led to the free-electron exchange approximation.

According to this approximation, the averaged exchange potential at point $\vec{r}$ is equal to the exchange potential for a free-electron gas whose total electronic charge density (both spins) is equal to that of the non-uniform system in question at the point $\vec{r}$ : 


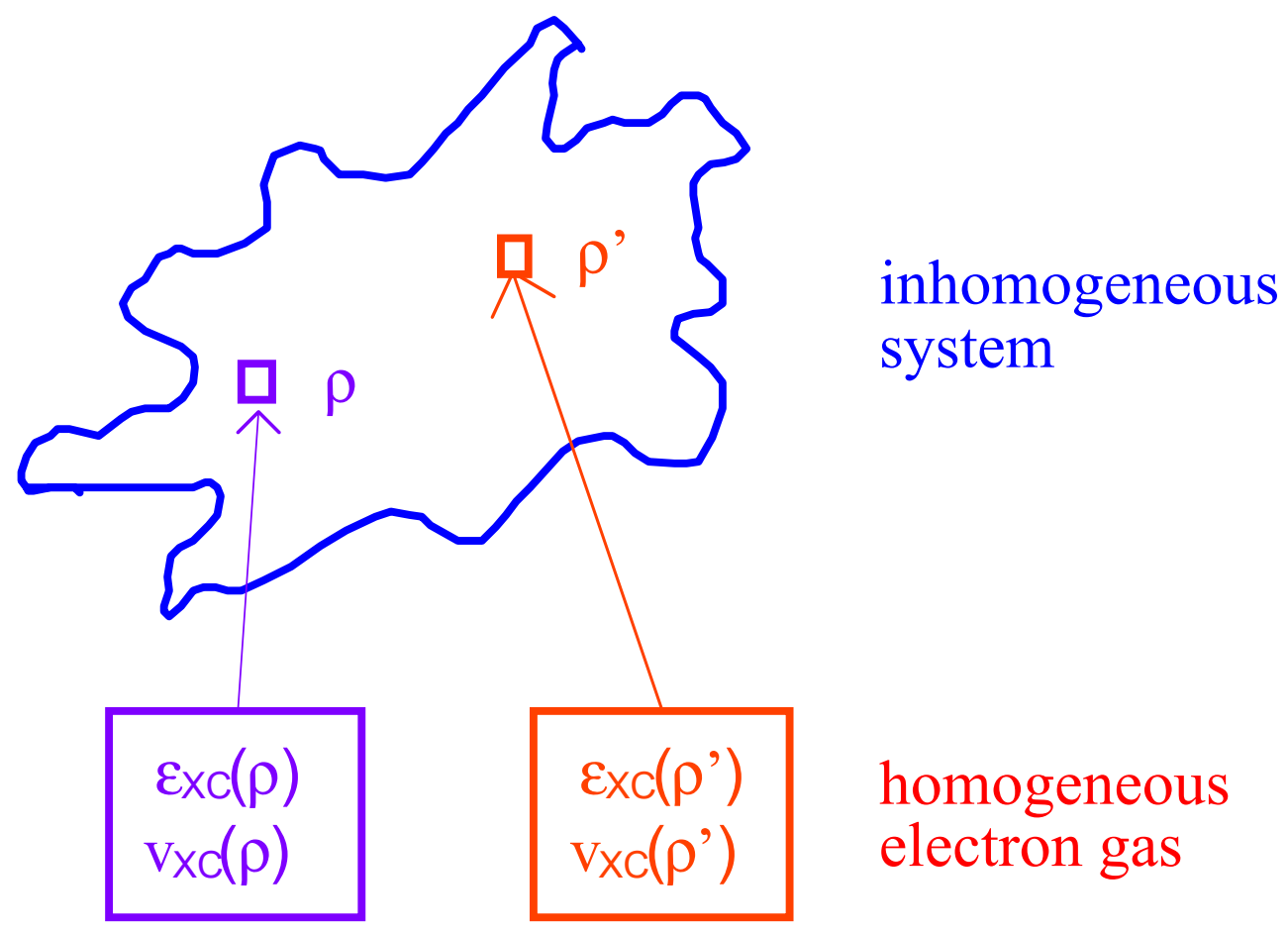

Thus, the averaged exchange potential becomes a function only of $\rho(r)$, and the problem of calculating exchange integrals is completely avoided.

Since the similarities between the exchange holes for a free-electron gas and an atomic system form the basis of the free-electron exchange approximation, our discussion thus far has been concerned only with these similarities.

\section{Modern functionals: $V_{X C}[\rho(r)]$}

There is a whole bunch of new functional i.e. LDA, GGA, meta-GGA, etc. ... available nowadays in the literature. ${ }^{[9-11]}$ The common feature of these functionals is that (i) they contain almost all of the dynamical correlation and (ii) that they are locally expressible. That is, for a spin-polarized case:

$$
V_{X C}\left[\rho_{\uparrow}(r), \rho_{\downarrow}(r)\right]=V_{X C}\left(\rho_{\uparrow}, \rho_{\downarrow}, \frac{d \rho \uparrow}{d r}, \frac{d \rho_{\downarrow}}{d r}, \frac{d^{2} \rho \uparrow}{d r^{2}}, \frac{d^{2} \rho_{\downarrow}}{d r^{2}}, \ldots\right)=V_{X C}(r)
$$

where $\rho \uparrow$ denote the density of electrons with respectively up and down spin. The term $\mathrm{V}_{\mathrm{XC}}$ is called exchange correlation potential. Here, $\mathrm{V}_{\mathrm{XC}}$ includes all the many particle interactions. Since the Hartree term and $V_{X C}$ depend on $\rho(r)$, which depends on the $\phi_{i}$, which in turn depend on $V$, the problem of solving the Kohn-Sham equation has to be done in a self-consistent way. In case of the free electron approximation, this term reads: 


$$
V_{X C}(r) \approx-6\left[\frac{3}{8 \pi} \rho(r)\right]^{1 / 3}
$$

\section{Atomic Kohn-Sham Equation}

Slater's original paper is concerned exclusively with a single electronic configuration composed only of closed shells. The many-electron wave function $\psi$ is represented by a single determinantal wave function built up from spinorbitals of the type (3). In this representation, the same radial wave function $R_{n} \lambda(r)$ is assigned to both sets of spins for any given orbital $n \lambda$, i.e,;

$$
R_{n \lambda \alpha}(r)=R_{n \lambda \beta}(r)=R_{n \lambda}(r)
$$

where the spin quantum number s is denoted by $\alpha$ for "up" spins, and by $\beta$ for "down" spins. Since there are equal numbers of up and down spin electrons in each orbital in a closed shell configuration, the electronic charge densities and the free-electron exchange potentials for the two sets of spins are equal.

A number of authors have extended Slater's work to encompass the case of an open shell configuration containing unequal numbers of up and down spins. In this extension, the manyelectron wave function is approximated by a single determinantal wave function built up from spinorbitals of the type (2). In this representation, different radial wave functions $R_{n \lambda \alpha}(r)$ and $R_{n \lambda \beta}(r)$ are assigned to the two sets of spins. This leads to different electronic charge densities and different free-electron exchange potentials for the two sets of spins. Although this representation does not treat the multiplet structure properly and violates certain symmetry requirements, it does provide a simple means for dealing with spin or exchange polarization, which is of interest in many magnetic problems.

In the present approach, we shall treat single electronic configurations having one or more open shell on the same basis as configurations having only closed shells. In all cases, the many-electron wave function will be represented by a single determinantal wave function built up from spinorbitals of the type (3). In contrast to the work just mentioned, we shall not concern ourselves with the distinctive features introduced by the presence of unmatched spins. More broadly, we shall ignore multiplet structure completely, and define an electronic configuration solely in terms of the occupation numbers of the various orbitals.

The radial Kohn-Sham wave equations for a free atom or ion can be written in the following form:

$$
\left\lfloor-\frac{d^{2}}{d r^{2}}+\frac{\lambda(\lambda+1)}{r^{2}}+V(r)\right\rfloor P_{n \lambda}(r)=E_{n \lambda} P_{n \lambda}(r)
$$


where $P_{n \lambda}(r)=r R_{n \lambda}(r)$ and $\mathrm{V}(\mathrm{r})$ is the sum of the nuclear coulomb potential, the total electronic coulomb potential, and the exchange potential. We shall tentatively set $\mathrm{V}(\mathrm{r})$ equal to $\mathrm{V}_{0}(\mathrm{r})$ at all values of $r$, where $V_{0}(r)$ is defined as follows:

$$
\mathrm{V}_{0}(\mathrm{r})=-\frac{2 \mathrm{Z}}{\mathrm{r}}+\left(\frac{2}{\mathrm{r}}\right) \int_{0}^{\mathrm{r}} \sigma(\mathrm{t}) \mathrm{dt}+2 \int_{\mathrm{r}}^{\infty} \frac{\sigma(\mathrm{t})}{\mathrm{t}} \mathrm{dt}-6\left[-\frac{3}{8 \pi} \rho(\mathrm{r})\right]^{1 / 3}
$$

$\mathrm{Z}$ is the atomic number, and $\rho(r)=\frac{\sigma(r)}{4 \pi r^{2}}$ is the spherically averaged total electronic charge density (both spins). Note that the electronic coulomb potential is expressed in terms of $\sigma(r)$, and the freeelectron exchange potential in terms of $\rho(r)$. Distances are measured in Bohr units and energies in Rydberg units.

The spherically averaged total electronic charge density is given by

$$
\rho(r)=\frac{\sigma(r)}{4 \pi r^{2}} ; \quad \sigma(r)=\sum_{n \lambda} \omega_{n \lambda}\left[P_{n \lambda}(r)\right]^{2}
$$

where $\omega_{n \lambda}$ is the occupation number for the orbital $n \lambda$ (both spins). In the special case of a closed shell, $\omega_{n \lambda}=2(2 \lambda+1)$. More generally, the total number of electrons is

$$
N=\sum_{n \lambda} \omega_{n \lambda}
$$

and the ionicity is:

$$
\text { qion }=\mathrm{Z}-\mathrm{N}
$$

The $\mathrm{P}_{\mathrm{n}} \lambda(\mathrm{r})$ are normalized radial wave functions:

$$
\int_{0}^{\infty}\left[P_{n \lambda}(r)\right]^{2} d r=1
$$

As noted in the previous section, the free-electron approximation breaks down at large distances from the nucleus. This can be seen directly by considering the wave equation (2) for a neutral atom. At sufficiently large values of $r$, the nuclear coulomb potential is exactly cancelled by the total electronic coulomb potential, and V(r) reduces to the free-electron exchange potential. Since the latter quantity depends on $\rho(r)$, which approaches zero as $r$ becomes large, $V(r)$ also approaches zero at large $r$. But this is manifestly incorrect. Since an electron cannot act on itself, it must move 
in the field of a singly charged positive ion at sufficiently large values of $r$. In other words, V(r) must approach $-\frac{2}{r}$, rather than zero, at large values of $r$.

In fact, the free-electron exchange approximation fails to treat the self-Coulomb potential properly at large values of $r$. In the conventional Hartree-Fock method, the self-coulomb potential term in the total electronic coulomb potential is exactly cancelled by a corresponding term (the self-exchange potential) in the total exchange potential. Since the free-electron exchange potential approaches zero as $r$ becomes large, the self-coulomb potential term is not cancelled out at large $r$. This leads to the incorrect behaviour of $V(r)$ at large distances.

In the present approach, we attempted to remedy this defect in the free-electron exchange approximation by following the well-known procedure of Latter. The essential idea of this procedure is to redefine $V(r)$ so that it has the correct asymptotic behaviour at large $r$. Let us consider an atom or ion with atomic number $Z$ containing $N$ electrons. Let the potential already defined by (6) again be denoted by $V_{0}(r)$, and let us call this the unmodified Kohn-Sham potential. Let us denote by $V(r)$ the potential that is actually introduced into the wave equation and let us call this the modified Kohn-Sham potential. We shall now define $V(r)$ in terms of $V_{0}(r)$ as follows:

$$
V(r)= \begin{cases}V_{0}(r) & \text { if } r<r_{0} \\ -\frac{2(Z-N+1)}{r} & \text { if } r \geq r_{0}\end{cases}
$$

where $\mathrm{r}_{0}$ is the value of $\mathrm{r}$ at which $V_{0}(r)=-\frac{2(Z-N+1)}{r}$, i.e.:

$$
V_{0}\left(r_{0}\right)=-\frac{2(Z-N+1)}{r_{0}}
$$

Thus, the free-electron exchange approximation is used only in the interior region, where it is satisfactory, and the self-coulomb potential is properly taken into account at large values of $r$. The behaviour of $V(r)$ at intermediate $r$ is of course subject to question, and the discontinuous behaviour of $\left(\frac{d V}{d r}\right)_{r=r_{0}}$ is distinctly "unphysical". In spite of its arbitrary character in the neighbourhood of $r=r_{0}$, the modified potential $V(r)$ is decidedly better than the unmodified potential $V_{0}(r)$.

\section{Description of the atomic multiplet structure}

If one neglects spin-orbit coupling, the multiplet wave functions $\Psi_{i}=\left|\alpha \Gamma m_{\Gamma} S m_{S}\right\rangle$ arising 
from a given configuration $\alpha$ are obtained by vector coupling as

$$
\Psi_{i}=\sum_{\mu} A_{i \mu} \Phi_{\mu}
$$

where $\Gamma$ is the label of the irreducible representation of the space part of the wave function, $\mathrm{m}_{\Gamma}$ refers to its component in case of degeneracy, $\mathrm{S}$ is the spin part of the wave function with component $\mathrm{m}_{\mathrm{S}}$ in case of spin multiplicity larger than $1 ; \Phi_{\mu}=\left|\chi_{1} \chi_{2} \chi_{3} \ldots\right|$ is a single-determinant wave function of spin-orbitals $\chi_{1}, \chi_{2}, \chi_{3}, \ldots$, and $\mathrm{A}_{\mathrm{i \mu}}$ is an orthogonal square matrix of symmetry coefficients. This vector-coupling task is best achieved automatically in a computer program. In case of a central field potential, the angular part of $\Psi_{\mathrm{i}}$ will be the well-known spherical harmonics $\mid \mathrm{LM}_{\mathrm{L}}>$ and we shall hence use this symbols instead. This notation will apply to all cas es considered in this work.

Let us note that DF or HF calculations provide directly the energies associated with the $\Phi_{\mu}$, which may be interpreted as barycenters of the various energies resulting from the states generated by the corresponding configuration. On the other hand, this energy $\mathrm{E}\left(\Phi_{\mu}\right)$ can also be obtained from Slater's rules for a single determinant as a sum of coulomb and exchange integrals (ignoring the constant one-electron part for the determinants of a single configuration):

$$
\begin{aligned}
\mathrm{E}\left(\Phi_{\mu}\right) & =\sum_{\mathrm{p}<\mathrm{q}}\left[\iint \chi_{\mathrm{p}}^{*}(1) \chi_{\mathrm{q}}^{*}(2) \mathrm{G}_{12} \chi_{\mathrm{p}}(1) \chi_{\mathrm{q}}(2) \mathrm{dV}_{1} \mathrm{dV} \mathrm{V}_{2}-\iint \chi_{\mathrm{p}}^{*}(1) \chi_{\mathrm{q}}^{*}(2) \mathrm{G}_{12} \chi_{\mathrm{p}}(2) \chi_{\mathrm{q}}(1) \mathrm{dV}_{1} \mathrm{dV}_{2}\right] \\
& =\sum_{\mathrm{p}<\mathrm{q}}\left[\left\langle\chi_{\mathrm{p}} \chi_{\mathrm{p}} \mid \chi_{\mathrm{q}} \chi_{\mathrm{q}}\right\rangle-\left\langle\chi_{\mathrm{p}} \chi_{\mathrm{q}} \mid \chi_{\mathrm{p}} \chi_{\mathrm{q}}\right\rangle\right] ; \text { where } \mathrm{G}_{12}=\frac{1}{\mathrm{r}_{12}}
\end{aligned}
$$

For cases where CI occurs similar expressions for off-diagonal electrostatic matrix elements are easily obtained and expressed in term of generalized two-electron integrals $\left\langle\chi_{p} \chi_{q} \mid \chi_{r} \chi_{s}\right\rangle$. In order to obtain these electrostatic integrals we need the spherical harmonics addition theorem and the Laplace expansion of $1 / \mathrm{r}_{12}$.

\section{The addition theorem for spherical harmonics}

The most useful property of the spherical harmonics is probably the reduction of products of spherical harmonics to linear combination of individual harmonics with the aid of Clebsch-Gordan series. That is, the so-called spherical addition theorem, which reads:

$$
Y_{l m}(\theta, \phi) Y_{l^{\prime} m^{\prime}}(\theta, \phi)=\sum_{M} C_{M m m^{\prime}}^{L l} l^{l} l^{\prime} Y_{L M}(\theta, \phi)
$$


with $\mathrm{M}=\mathrm{m}+\mathrm{m}^{\prime}$. We may regard the coupling coefficient $C_{M m m^{\prime}}^{L l}$, also called Clebsch-Gordan coefficient, as being defined in the appearance of this equation. Hence, if we multiply both sides of this equation by $Y_{L M}^{*}(\theta, \phi)$, integrate over unity sphere and use the orthogonality of the spherical harmonics, we obtain

$$
C_{M m m^{\prime}}^{L l l^{\prime}}=\int d \Omega Y_{L M}^{*}(\theta, \phi) Y_{l m}(\theta, \phi) Y_{l^{\prime} m^{\prime}}(\theta, \phi)
$$

This is obviously not the only possibility to calculate these coupling coefficients. Usually the numerical evaluation of them is carried out by computer programs, which are easy to develop or to obtain.

In group theory, this is exactly equivalent to the reduction of a reducible representation obtained by taking the direct product of two irreducible representations $\Gamma$, i.e.

$$
\Gamma \otimes \Gamma=\sum_{i} \Gamma_{i}
$$

\section{Expansion of $1 / r_{12}$}

In terms of the distances $r_{1}$ and $r_{2}$ and the angle $g$ between the two vectors from the origin to the two particles, the distance $r_{12}$ between the two particles is:

$$
r_{12}=\sqrt{r_{1}^{2}+r_{2}^{2}-2 r_{1} r_{2} \cos (\gamma)}
$$

if we let $r_{>}$be the greater of $r_{1}$ and $r_{2}$, and $r_{<}$be the lesser, then

$$
r_{12}=r_{>} \sqrt{1+x^{2}-2 x \cos (\gamma)}
$$

where $x=\frac{r_{<}}{r_{>}}$. The expansion of this expression in term of Legendre polynomials yields

$$
\frac{1}{r_{12}}=\frac{1}{r_{>}} \sum_{n} x^{n} P_{n}(\cos \gamma)=\sum_{n} \frac{r_{<}^{n}}{r_{>}^{n+1}} P_{n}(\cos \gamma)
$$

We must now express $P_{n}(\cos \gamma)$ as a function of the spherical coordinates - the polar and the azimuthal angles $\theta$ and $\phi$ - of the two particles. Considering this as a function of $\theta_{1}$ and $\phi_{1}$, we may expand it in terms of the orthogonal functions: $Y_{n m}\left(\theta_{1}, \phi_{1}\right)=P_{n}^{|m|}\left(\cos \theta_{1}\right) e^{i m \phi_{1}}$, i.e. the so-called spherical harmonics, which are expressed in terms of the associated Legendre functions $P_{n}^{|m|}(x)$. $P_{n}(\cos \gamma)$ is a solution of the angular part of the atomic Schrödinger eq. 


$$
\frac{1}{\sin \theta_{1}} \frac{\partial}{\partial \theta_{1}}\left(\sin \theta_{1} \frac{\partial P_{n}}{\partial \theta_{1}}\right)+\frac{1}{\sin ^{2} \theta_{1}} \frac{\partial^{2} P_{n}}{\partial \phi_{1}^{2}}+n(n+1) P_{n}=0
$$

since this eq. remains unchanged under any rotation. The general solution of this eq. is a linear combination of the spherical harmonics $\mathrm{Y}_{\mathrm{nm}}$, so we may express $P_{n}(\cos \gamma)$ as

$$
P_{n}(\cos \gamma)=\sum_{m=-n}^{m=n} A_{n m} P_{n}^{|m|}\left(\cos \theta_{1}\right) e^{i m \phi_{1}}
$$

The same must hold for the second particle as well. Thus, using the orthogonality of the Legendre functions, it is possible to show that

$$
A_{n m}=\frac{2 \pi}{2 n+1} P_{n}^{|m|}\left(\cos \theta_{2}\right) e^{-i m \phi_{2}}
$$

Thus, we get the final result

$$
\frac{1}{r_{12}}=\sum_{n} \frac{2 \pi}{2 n+1} \frac{r_{<}^{n}}{r_{>}^{n+1}} \sum_{m=-n}^{m=n} Y_{n m}\left(\theta_{1}, \phi_{1}\right) Y_{n m}^{*}\left(\theta_{2}, \phi_{2}\right)
$$

\section{Electrostatic two-electron integrals}

It is now easy to express the sought two-electron integrals in terms of a limited number of reduced electrostatic matrix elements as follows. Consider a specific two-electron integral

$$
\langle a b \mid c d\rangle=\left\langle R_{a}\left(r_{1}\right) Y_{l_{a} m_{a}}\left(\theta_{1}, \phi_{1}\right) R_{b}\left(r_{2}\right) Y_{l_{b} m_{b}}\left(\theta_{2}, \phi_{2}\right)\left|G_{12}\right| R_{c}\left(r_{1}\right) Y_{l_{c} m_{c}}\left(\theta_{1}, \phi_{1}\right) R_{d}\left(r_{2}\right) Y_{l_{d} m_{d}}\left(\theta_{2}, \phi_{2}\right)\right\rangle
$$

one obtains, first by expanding $1 / \mathrm{r}_{12}$ as above and next by vector coupling of the orbitals a and $\mathrm{c}$ belonging to electron 1 and the orbitals $\mathrm{b}$ and $\mathrm{d}$ belonging to electron 2 ,

$$
\left\langle a(1) b(2)\left|G_{12}\right| c(1) d(2)\right\rangle=\sum_{k m} \frac{4 \pi}{2 k+1}\left\langle R_{a}(1) R_{b}(2)\left|\frac{r_{<}^{k}}{r_{>}^{k+1}}\right| R_{c}(1) R_{d}(2)\right\rangle C\left(\begin{array}{ccc}
l_{a} & l_{c} & k \\
m_{a} & m_{c} & m
\end{array}\right) C\left(\begin{array}{ccc}
l_{b} & l_{d} & k \\
m_{b} & m_{d} & m
\end{array}\right)
$$

where

$$
\begin{aligned}
\frac{4 \pi}{2 k+1}\left\langle R_{a}(1) R_{b}(2)\left|\frac{r_{<}^{k}}{r_{>}^{k+1}}\right| R_{c}(1) R_{d}(2)\right\rangle & =\frac{4 \pi}{2 k+1} \int \frac{r_{<}^{k}}{r_{>}^{k+1}} R_{a}\left(r_{1}\right) R_{b}\left(r_{2}\right) R_{c}\left(r_{1}\right) R_{d}\left(r_{2}\right) r_{1}^{2} d r_{1} r_{2}^{2} d r_{2} \\
& =W_{k}(a, b, c, d)
\end{aligned}
$$

are reduced two-electron electrostatic matrix elements (Racah or Slater-Condon parameters). 
The coupling coefficients $C_{m m^{\prime} M}^{l l^{\prime} K}$ have been mentioned afore and the final expression for a general electrostatic matrix elements reads:

$$
\langle a b \mid c d\rangle=\sum_{k m} C\left(\begin{array}{ccc}
l_{a} & l_{c} & k \\
m_{a} & m_{c} & m
\end{array}\right) C\left(\begin{array}{ccc}
l_{b} & l_{d} & k \\
m_{b} & m_{d} & m
\end{array}\right) W_{k}(a, b, c, d)
$$

The calculation of $\mathrm{W}_{\mathrm{k}}(\mathrm{a}, \mathrm{b}, \mathrm{c}, \mathrm{d})$ can be done either by numerical quadrature whenever the radial wave functions are given numerically ${ }^{[12]}$ or analytically when the wave function are expanded in a basis set. A computer program is given in the appendix for the case that the wave function is expanded in Slater type orbitals.

Thus, if we set the origin of energy equal to the configurational barycenter which is equivalent to the neglect of the constant one-electron part, the determinant energies $\mathrm{E}\left(\Phi_{\mu}\right)$ are related to Coulomb and exchange integrals which are in turn related to the reduced matrix elements $\mathrm{W}_{\mathrm{k}}(\mathrm{a}, \mathrm{b}, \mathrm{c}, \mathrm{d})$. Combining these 2 equations yields

$$
E\left(\Phi_{\mu}\right)=\sum_{k} B_{\mu k} W_{k}
$$

where $\mathrm{W}_{\mathrm{k}}$ are the reduced electrostatic matrix elements discussed earlier and $\mathrm{B}_{\mu \mathrm{k}}$ is a rectangular matrix of expansion coefficients. The dimension of th is matrix $\mathbf{B}$ is $\mathrm{m} \times \mathrm{n}$, where $\mathrm{m}$ is the number of single determinants or micro-states within the set of configurations considered and $\mathrm{n}$ is the number of all reduced electrostatic matrix elements occurring within the full space of spin-orbitals $\chi_{1}, \chi_{2}$, $\chi_{3}, \ldots$ composing the micro-states. Note that $\mathrm{n} \leq \mathrm{m}$ for all systems.

Example: The calculation of all multiplets of a $d^{2}$ configuration of a free atomic ion

The configuration $\mathrm{d}^{2}$ of a free metal ion generates the following five states: ${ }^{1} \mathrm{~S},{ }^{3} \mathrm{P},{ }^{1} \mathrm{D},{ }^{3} \mathrm{~F}$ and ${ }^{1} \mathrm{G}$. There are 45 symmetry-adapted N-electron wave functions $\Psi_{i}$ and 45 micro-states $\Phi_{\mu}$. In order to save lengthy tabulations let us skip the intermediate steps we have already illustrated with details in the two previous examples and give immediately the reduced electrostatic matrix elements:

$$
\begin{aligned}
& R_{1}=R(d d ; d d ; S)=5 \cdot J\left(d_{x y}, d_{x y}\right)-12 \cdot K\left(d_{x y}, d_{y z}\right)+4 \cdot K\left(d_{x y}, d_{z} 2\right) \\
& R_{2}=R(d d ; d d ; D)=-6 \cdot K\left(d_{x y}, d_{y z}\right)+8 \cdot K\left(d_{x y}, d_{z} 2\right) \\
& R_{3}=R(d d ; d d ; G)=8 \cdot K\left(d_{x y}, d_{y z}\right)-6 \cdot K\left(d_{x y}, d_{z} 2\right)
\end{aligned}
$$


where $\mathrm{J}$ and $\mathrm{K}$ denote respectively Coulomb and exchange integrals. In the present case, these reduced electrostatic matrix elements are identical to Slater-Condon parameters, whereas the nonredundant determinants are:

$$
\Phi_{1}=\left|\bar{d}_{x y}^{+} \bar{d}_{x y}\right| ; \quad \Phi_{2}=\left|\bar{d}_{x y} \bar{d}_{y z}\right| ; \quad \Phi_{3}=\left|\bar{d}_{z^{2}}^{+} \bar{d}_{x y}\right|
$$

Thus, in this case, our scheme induces the calculation of only three excited single determinants within the 45 possible on es. The 42 remaining single determinants are redundant and their energies are expressible as linear combinations of the three former ones. The values of $\mathrm{W}_{\kappa}$ are :

$$
\left(\begin{array}{l}
W_{1} \\
W_{2} \\
W_{3}
\end{array}\right)=\left(\begin{array}{ccc}
7 / 3 & 4 & -4 / 3 \\
2 / 3 & 2 & -8 / 3 \\
2 / 3 & -8 / 3 & 2
\end{array}\right)\left(\begin{array}{l}
E\left(\Phi_{1}\right) \\
E\left(\Phi_{2}\right) \\
E\left(\Phi_{3}\right)
\end{array}\right)
$$

Following the procedure outlined earlier we get finally the state energies $\mathrm{E}\left(\Psi_{\mathrm{i}}\right)$ as:

$$
\left(\begin{array}{l}
E\left({ }^{1} S\right) \\
E\left({ }^{3} P\right) \\
E\left({ }^{1} D\right) \\
E\left({ }^{3} F\right) \\
E\left({ }^{1} G\right)
\end{array}\right)=\left(\begin{array}{ccc}
7 / 3 & -2 & 2 / 3 \\
5 & 0 & -4 \\
2 / 3 & -1 & 4 / 3 \\
0 & 0 & 1 \\
2 / 3 & 4 / 3 & -1
\end{array}\right)\left(\begin{array}{l}
E\left(\left|d_{x y}^{+} d_{x y}{ }^{-}\right|\right) \\
E\left(\mid d_{x y}^{-}-d_{y z}\right) \\
E\left(\left|d_{z^{2}}^{+} d_{x y}\right|\right)
\end{array}\right)
$$

We have thus expressed the energy of the five multiplets in terms of the energy of three nonredundant single determinants each of them being obtained in a corresponding DF calculation.

\section{Energy of single determinants}

\section{Step 1:}

Solve the KS equation for the symmetrically averaged density of all ground and excited state configurations in separate SCF calculations. The obtained KS orbitals for each configuration are kept frozen for the next step. For example, when $\alpha=t_{2}^{2} e^{1}$, the occupations of the $t_{2}$ orbitals are $n_{\xi^{\uparrow}}=n_{\xi^{\downarrow}}=n_{\eta^{\uparrow}}=n_{\eta^{\downarrow}}=n_{\zeta^{\uparrow}}=n_{\zeta^{\downarrow}}=\frac{1}{3}$ and those of the e orbitals are $n_{\theta^{\uparrow}}=n_{\theta^{\downarrow}}=n_{\mathcal{E}^{\uparrow}}=n_{\mathcal{E}^{\downarrow}}=\frac{1}{4}$.

\section{Step 2:}

Get the energy $\mathrm{E}(\Phi)$ of all needed single determinants arising from the corresponding configuration. It should be stressed that the calculation of single determinants must yield a totally symmetric 
electron density. This is not the case if the orbitals belonging to a degenerate irreducible representation (irrep) in the considered point group are not totally occupied in the single determinant. Thus, the symmetry has to be lowered to a point group where the degenerate irrep splits, to that the condition of the totally electron density is fulfilled.

Step 3:

From these energies i.e. $\mathrm{E}(\Phi)$, get the multiplet energies according to section: "Description of the atomic multiplet structure". Excitation energies are finally calculated by taking the differences between ground state and excited state multiplet energies.

The second step has to be repeated $r$ times, i.e. as many times as there are non-redundant determinants in that configuration. It should be pointed out that this procedure does not always fulfill exactly Eq. (37), the redundancy condition for single determinants. The discrepancy may be as large as several tenths of an $\mathrm{eV}$. In these cases we suggest for example to calculate the energies of all possible single determinants $\Phi_{\mu} ; \mu=1,2, \ldots, \mathrm{m}$ and to take the least squares average of them:

$$
\left(\begin{array}{c}
E\left(\Phi_{\mu_{1}}\right) \\
\ldots \\
E\left(\Phi_{\mu_{i}}\right) \\
\cdots \\
E\left(\Phi_{\mu_{r}}\right)
\end{array}\right)=\left(\mathbf{C}^{\mathbf{T}} \mathbf{C}\right)^{-1} \mathbf{C}^{\mathbf{T}}\left(\begin{array}{c}
E\left(\Phi_{1}\right) \\
\ldots \\
E\left(\Phi_{\mu}\right) \\
\cdots \\
E\left(\Phi_{m}\right)
\end{array}\right)
$$

For the sake of completeness, it should be poin ted out that, especially, in cases of ions with multiple open shells the occurrence of states with identical spin space symmetry is increasingly frequent. In those cases, obviously, configuration interaction should also be taken into account. However, the calculation of the off-diagonal electrostatic matrix element are readily obtained from the radial integrals and do not present a great difficulty.

\section{Energy of a n atomic density}

The diagonal two-particle density matrix is

$$
\rho_{2}\left(\vec{r}_{1}, \vec{r}_{2}\right)=\frac{N(N-1)}{2} \int \ldots \int\left|\Psi\left(\vec{r}_{1}, \vec{r}_{2}, \vec{r}_{3}, \ldots\right)\right|^{2} d \vec{r}_{3} \ldots d s_{1} \ldots
$$

The factors $\frac{N(N-1)}{2}$ in front are the number of equivalent pairs. 
Similarly the one-particle density matrices are

$$
\begin{aligned}
& \rho_{1}\left(\vec{r}_{1}, \vec{r}_{1}^{\prime}\right)=N \int \ldots \int \Psi^{*}\left(\vec{r}_{1}^{\prime}, \vec{r}_{2}, \vec{r}_{3}, \ldots\right) \Psi\left(\vec{r}_{1}, \vec{r}_{2}, \vec{r}_{3}, \ldots\right) d \vec{r}_{2} \ldots d s_{1} \ldots \\
& \rho_{1}\left(\vec{r}_{1}\right)=N \int \ldots \int\left|\Psi\left(\vec{r}_{1}, \vec{r}_{2}, \vec{r}_{3}, \ldots\right)\right|^{2} d \vec{r}_{2} \ldots d s_{1} \ldots
\end{aligned}
$$

The last equation gives the usual electron density $\rho(\vec{r})=\rho_{1}(\vec{r})$.

The energy E, given as the expectation value of the Hamiltonian is expressed in terms of these matrices,

$$
E=\int\left[-\frac{1}{2} \nabla_{\vec{r}}^{2} \rho_{1}\left(\vec{r}^{\prime}, \vec{r}\right)\right]_{\vec{r}^{\prime}=\vec{r}} d \vec{r}+\int v(\vec{r}) \rho_{1}(\vec{r}) d \vec{r}+\iint \frac{\rho_{2}\left(\vec{r}_{1}, \vec{r}_{2}\right)}{\left|\vec{r}_{1}-\vec{r}_{2}\right|} d \vec{r}_{1} d \vec{r}_{2}
$$

In Hartree-Fock or Kohn-Sham theory the one-particle density matric es are represented in terms of orbitals:

$$
\begin{aligned}
& \rho_{1}\left(\vec{r}_{1}^{\prime}, \vec{r}_{1}\right)=\sum_{i} v_{i} \phi_{i}^{*}\left(\vec{r}_{1}^{\prime}\right) \phi_{i}\left(\vec{r}_{1}\right) \\
& \rho_{1}\left(\vec{r}_{1}\right)=\sum_{i} v_{i}\left|\phi_{i}\left(\vec{r}_{1}\right)\right|^{2}
\end{aligned}
$$

In Hartree-Fock theory, the exchange energy is

$$
K=-\iint \frac{\phi_{i}^{*}\left(\vec{r}_{1}\right) \phi_{i}\left(\vec{r}_{2}\right) \phi_{j}^{*}\left(\vec{r}_{2}\right) \phi_{j}\left(\vec{r}_{1}\right)}{\left|\vec{r}_{1}-\vec{r}_{2}\right|} d \vec{r}_{1} d \vec{r}_{2}=-\frac{1}{4} \iint \frac{\left|\rho_{1}\left(\vec{r}_{1}, \vec{r}_{2}\right)\right|^{2}}{\left|\vec{r}_{1}-\vec{r}_{2}\right|} d \vec{r}_{1} d \vec{r}_{2}
$$

\section{Energies of a tomic micro-states}

Consider: $\phi_{i}(\vec{r})=\varphi_{i}(\vec{r}) \sigma_{i}(s)$ KS spinorbitals and $v_{\mathrm{i}}=0$ or 1 the corresponding occupations.

Thus $\rho(\vec{r})=\sum_{i} v_{i} \phi_{i}^{2}(\vec{r})$ is the density corresponding to the single det. $|\Phi\rangle=\mid \ldots \phi_{i} \ldots ; \forall i$ with $v_{i}=1$

$$
\begin{aligned}
E[\rho] & =\sum_{i=1} v_{i}\left\langle\phi_{i}\left|-\frac{1}{2} \nabla^{2}\right| \phi_{i}\right\rangle+\sum_{i=1} v_{i}\left\langle\phi_{i}\left|-\frac{Z_{A}}{r_{A}}\right| \phi_{i}\right\rangle+\frac{1}{2} \sum_{i j} v_{i} v_{j}\left\langle\phi_{i}(1) \phi_{i}(1)\left|\frac{1}{r_{12}}\right| \phi_{j}(2) \phi_{j}(2)\right\rangle+\int \varepsilon_{X C}\left[\rho_{\uparrow}, \rho_{\downarrow}\right] \rho(\vec{r}) d \vec{r} \\
& =\sum_{i=1} v_{i} h_{i i}^{\text {core }}+\frac{1}{2} \sum_{i j} v_{i} v_{j} J_{i j}+\int \varepsilon_{X C}\left[\rho_{\uparrow}, \rho_{\downarrow}\right] \rho(\vec{r}) d \vec{r}
\end{aligned}
$$

On the other hand the KS spinorbitals satisfy: 


$$
\left\lfloor-\frac{1}{2} \nabla^{2}-\frac{Z}{r}+\int \frac{\rho\left(\vec{r}^{\prime}\right)}{\left|\vec{r}-\vec{r}^{\prime}\right|} d \vec{r}^{\prime}+v_{x c}(\vec{r})\right\rfloor \phi_{i}(\vec{r})=\varepsilon_{i} \phi_{i}(\vec{r}) \text {; where: } v_{x c}(\vec{r})=\frac{\delta E_{X C}[\rho]}{\delta \rho(\vec{r})}
$$

Ergo:

$$
\begin{aligned}
\varepsilon_{i} & =\left\langle\phi_{i}\left|-\frac{1}{2} \nabla^{2}\right| \phi_{i}\right\rangle+\left\langle\phi_{i}\left|-\frac{Z_{A}}{r_{A}}\right| \phi_{i}\right\rangle+\sum_{j} v_{j} J_{i j}+\left\langle\phi_{i} \mid v_{X C}\left[\rho_{\uparrow}, \rho_{\downarrow}\right] \phi_{i}\right\rangle \\
& =h_{i i}^{\text {core }}+\sum_{j} v_{j} J_{i j}+\left\langle\phi_{i}\left|v_{X C}\left[\rho_{\uparrow}, \rho_{\downarrow}\right]\right| \phi_{i}\right\rangle
\end{aligned}
$$

Thus,

$$
\begin{aligned}
E[\rho] & =\sum_{i=1} v_{i} h_{i i}^{\text {core }}+\frac{1}{2} \sum_{i j} v_{i} v_{j} J_{i j}+\int \varepsilon_{X C}\left[\rho_{\uparrow}, \rho_{\downarrow}\right] \rho(\vec{r}) d \vec{r} \\
& =\sum_{i=1} v_{i} \varepsilon_{i}-\frac{1}{2} \sum_{i j} v_{i} v_{j} J_{i j}+\int\left\{\varepsilon_{X C}\left[\rho_{\uparrow}, \rho_{\downarrow}\right]-v_{X C}\left[\rho_{\uparrow}, \rho_{\downarrow}\right]\right\} \rho(\vec{r}) d \vec{r}
\end{aligned}
$$

For an homogeneous electron gas of density $\rho$ :

$$
\varepsilon_{x}(\rho)=-C_{X} \rho^{1 / 3} \text { and } v_{x}(\rho)=-\frac{4}{3} C_{X} \rho^{1 / 3} ; \text { with } C_{X}=\frac{3}{4}\left(\frac{3}{\pi}\right)^{1 / 3}=0.7386
$$

In spin-polarized cases:

$$
E_{X}\left[\rho_{\uparrow}, \rho_{\downarrow}\right]=-2^{1 / 3} C_{X} \int\left[\left(\rho_{\uparrow}\right)^{4 / 3}+\left(\rho_{\downarrow}\right)^{4 / 3}\right] \overrightarrow{l r}
$$

and

$$
\left\langle\phi_{i} \mid v_{X C}\left[\rho_{\uparrow}, \rho_{\downarrow}\right] \phi_{i}\right\rangle=\left\{\begin{array}{l}
-\frac{4}{3} C_{X}\left\langle\phi_{i}\left|\rho_{\uparrow}^{1 / 3}\right| \phi_{i}\right\rangle \text { if } \uparrow \in i \\
-\frac{4}{3} C_{X}\left\langle\phi_{i}\left|\rho_{\downarrow}^{1 / 3}\right| \phi_{i}\right\rangle \text { if } \downarrow \in i
\end{array}\right.
$$

Hence

$$
\begin{aligned}
\int\left\{\mathcal{E}_{X C}\left[\rho_{\uparrow}, \rho_{\downarrow}\right]-v_{X C}\left[\rho_{\uparrow}, \rho_{\downarrow}\right]\right\} \rho(\vec{r}) d \vec{r} & =-2^{1 / 3} C_{X} \int\left[\left(\rho_{\uparrow}\right)^{4 / 3}+\left(\rho_{\downarrow}\right)^{4 / 3}\right] \vec{r}+\frac{4}{3} C_{X} \int\left[\left(\rho_{\uparrow}\right)^{4 / 3}+\left(\rho_{\downarrow}\right)^{4 / 3}\right] \vec{r} \\
& =\left(\frac{4}{3}-2^{1 / 3}\right) C_{X} \int\left[\left(\rho_{\uparrow}\right)^{4 / 3}+\left(\rho_{\downarrow}\right)^{4 / 3}\right] \vec{r}=0.0734 C_{X} \int\left[\left(\rho_{\uparrow}\right)^{4 / 3}+\left(\rho_{\downarrow}\right)^{4 / 3}\right] d \vec{r} \\
& =0.0542 \int\left[\left(\rho_{\uparrow}\right)^{4 / 3}+\left(\rho_{\downarrow}\right)^{4 / 3}\right] / \vec{r}
\end{aligned}
$$

19 
Finally

$$
\begin{aligned}
E[\rho] & =\sum_{i=1} v_{i} \varepsilon_{i}-\frac{1}{2} \sum_{i j} v_{i} v_{j} J_{i j}+\int\left\{\varepsilon_{X C}\left[\rho_{\uparrow}, \rho_{\downarrow}\right]-v_{X C}\left[\rho_{\uparrow}, \rho_{\downarrow}\right]\right\} \rho(\vec{r}) d \vec{r} \\
& \approx \sum_{i=1} v_{i} \varepsilon_{i}-\frac{1}{2} \sum_{i j} v_{i} v_{j} J_{i j}+0.0542 \int\left[\left(\rho_{\uparrow}\right)^{4 / 3}+\left(\rho_{\downarrow}\right)^{4 / 3}\right] d \vec{r}
\end{aligned}
$$

\section{Computational details}

In general, all DFT computations were performed using ADF 2004.01. ${ }^{[13]}$ Spherically symmetric ions were calculated as Averages Over Configurations (AOC), that is with equal, and generally fractional, occupations of all metallic orbitals. We used the same high-accuracy numerical settings as for an ADF fragment creation run (INTEGRATION keyword 10.0, SCF convergence criterion $10^{-8}$ ). We used the Volko-Wilk-Nusair ${ }^{[14]}$ (VWN) for the local density approximation and the Perdew-Wang ${ }^{[15]}$ non-local corrections (PW91) for the exchange and correlation functionals. Relativistic effects were taken into account using the scalar ZORA ${ }^{[16]}$ approximation. The basis set was ZORA/QZ4P (all electrons, quintuple zeta + polarization). The radial part of the Slater-type orbitals was extracted from the output files using custom scripts and the DMPKF utility, and subsequently used to evaluate the Slater integrals $F^{k}(k=0,2,4$ and 6$)$ using the method described in ref. [12] For comparison purposes, these integrals were transformed into the equivalent Racah parameters $B$ and $C$ for the $d$ metal ions and the reduced $F_{k}$ parameters for the lanthanides using the known linear combinations:

$$
\begin{gathered}
\left.\begin{array}{c}
B=1 / 49 F^{2}-5 / 441 F^{4} \\
C=35 / 441 F^{4} \\
F_{0}=F^{0} \\
F_{2}=F^{2} / 49 \\
F_{4}=F^{4} / 441
\end{array}\right\} d^{n} \\
\left.\begin{array}{c}
F_{0}=F^{0} \\
F_{2}=F^{2} / 225 \\
F_{4}=F^{4} / 1089 \\
F_{6}=F^{6} / 7361.64
\end{array}\right\} f^{n}
\end{gathered}
$$


The PW91 AOC results were also used to calculate the energies of all possible $\mathrm{d}^{n}$ and $\mathrm{f}^{n}$ Slater determinants. Electron repulsion parameters were then fitted to those energies as usual in the LFDFT method.

For lanthanide(III) ions, we performed further calculations using the LDA X-alpha approximation ${ }^{[8 \text {, }}$ ${ }^{17]}$ and the Van Leeuwen-Baerends ${ }^{[18]}$ (LB94) GGA functional. Convergence was only achieved for a few ions using the Becke-Perdew ${ }^{[19,20]}$ (BP86) GGA functional. We also include the results obtained from a fit of the Slater determinant energies using the VWN LDA functional.

\section{Results and discussion}

The calculated electron repulsion parameters are reported in Tables 1 and 2 . When available, the experimental values for the free ion are included. For the lanthanide(III) ions, where the f electrons are efficiently shielded from the ligand field, the aqua ions provide good estimates when the free ion values are missing as can be seen from the $\operatorname{Pr}^{3+}$ and $\mathrm{Er}^{3+}$ examples.

As a general rule, all calculated Slater integrals $F_{k}$ are within $30 \%$ of the experimental value. The only exceptions are the $F_{6}$ of the lanthanide(III) ions calculated from single determinant energies. The latter are systematically $70-85 \%$ lower than the experimental results. For any given series $\left(1^{\text {st }}\right.$ row divalent ions, $1^{\text {st }}$ row trivalent, $2^{\text {nd }}$ row divalent, .... $3^{\text {rd }}$ row trivalent and lanthanides), the theoretical $F_{k}$ increase almost linearly with the atomic number. This trend is in contrast with the dispersion of the experimental data points. The theoretical values are largely insensitive to the actual density functional, and the LDA and GGA results frequently differ by less than $1 \%$. It is therefore sufficient to choose one functional as a reference (PW91) and the associated comments can be taken as valid in all cases. The only significant division within our results is between the values obtained from the radial part of the wave function and those fitted to the Slater determinant energies, which we will discuss separately.

The Slater integrals $F_{k}$ calculated from the radial part of the wave function are generally overestimated in comparison with the experimental data, especially for the lower $k$ 's. For the $\mathrm{d}^{\mathrm{n}}$ ions of the first transition series, the theoretical $F_{2}$ 's are 7 to $23 \%$ higher than the experimental estimations. The relative difference between the calculated and experimental $F_{4}$ is between -4 and $+22 \%$. The empirical ratio $B / C=4^{[21]}$ is reproduced in the theoretical values. For the second and third series, the experimental data is seriously incomplete, which makes a systematic comparison difficult. However, based on the available results, the same trends can be noticed. The theoretical Slater integrals are still larger than their few experimental counterparts. Interestingly, the ratio $B / C \sim 4$ is retained in our calculations for the heavier transition metal ions, but not in the experimental data $\left(2.2\right.$ for $\mathrm{Nb}^{3+}, 6.5$ for $\mathrm{Rh}^{3+}$ ). The experimental data for the lanthanide(III) ions is 
exhaustive in aqueous solution, ${ }^{[22-25]}$ which makes the $\mathrm{f}^{n}$ excellent standards for the calibration of theoretical calculations. Here again, the electron repulsion parameters calculated for the gas phase are larger than those obtained experimentally, but we observe a steady improvement along the series for all parameters $\left(F_{2}+37 \%\right.$ for $\operatorname{Pr}^{3+}$ vs. $+24 \%$ for $\mathrm{Tm}^{3+} ; F_{4}+17 \%$ vs. $4 \%, F_{6}+30 \%$ vs. $+1 \%$ ). The calculated $F_{4} / F_{2}$ ratio is 0.129 , slightly lower than the average experimental value of 0.15 but consistent with the Hartree-Fock and Dirac-Fock results of Freeman et al. ${ }^{[26,27]}$ This is of course due to the more significant overestimation of the $2^{\text {nd }}$ order integral $F_{2}$ in our calculations. The same is true for the $F_{6} F_{2}$ ratio, with an average calculated value of 0.0137 (experimental $0.015^{[22]}, \mathrm{H}-\mathrm{F} 0.0139^{[26]}$ ).

The Slater integrals obtained from a fit of the single determinant energies are always lower than those calculated from the radial part of the wave function. For the early divalent ions of the first transition series (from $\mathrm{V}^{2+}$ to $\mathrm{Mn}^{2+}$ ), they are slightly higher than the experimental values ( 0 to $5 \%$ for $F_{2},-7$ to $+4 \%$ for $F_{4}$ ). This is also the case for $F_{2}$ in the lanthanide(III) ions (14 to $26 \%$ ). In all other cases, the calculated value is lower than the experimental one. As noted earlier, the lanthanide $F_{6}$ are most strongly affected as they are underestimated by almost one order of magnitude. The difference between the calculated and experimental lanthanide $F_{4}$ is not as severe, but still significant ( -14 to $-28 \%$ along the series). For the trivalent ions of the first transition series, the calculated Slater integrals are lower than the experimental values regardless of $k$. The $2^{\text {nd }}$ and $3^{\text {rd }}$ transition series ions show the same trend when comparing the radial wave function results to those of single determinant energy calculations.

As a consequence of the low $F_{4}$ and $F_{6}$, the lanthanide $F_{k} / F_{2}$ ratios $(k=4,6)$ calculated from SD energies differ significantly from their experimental and radial wavefunction-based equivalents. This is already clear for the $4^{\text {th }}$ order parameter $\left(F_{4} / F_{2}<0.1\right)$, but much more striking for the $6^{\text {th }}$ order integral $\left(F_{6} / F_{2} \approx 0.0016\right)$.

The clear difference between the Slater integrals calculated from the radial wave functions and those fitted to SD energies can probably be assigned to a systematic error in the SD DFT calculations. The relative energies of the single determinants will be determined essentially by the Coulomb and exchange contributions of the electrostatic energy term. In standard DFT, the exchange part can only be approximated by an ad hoc functional of the LDA, GGA or meta-GGA type. In order to solve that problem, hybrid functionals such as $\mathrm{B} 3 \mathrm{LYP}^{[28]}$ have been introduced. Unfortunately, such functional have only recently become available in ADF and at the time of this writing their use is limited to spin-restricted calculations.

A possible explanation for the overestimation of the integrals in our radial wavefunction-based results could be a bias of the experimental data due to the nephelauxetic effect. Well established for the $d$ elements, ${ }^{[29-31]}$ that effect is sometimes invoked in the discussion of lanthanide 
spectroscopy. ${ }^{[32,33]}$

Another problem in the calculation could be the self-correction energy. ${ }^{[34]}$ An exact functional must be self-interaction free, which is not the case for the LDA and GGA approximations used in this work. Self-interaction corrections can be added to DFT codes ${ }^{[35]}$ such as ADF. However, the current level of support is not sufficient to allow a systematic study. In particular, the implementation found in $\mathrm{ADF} 2004.01$ is not suitable for calculations involving $\mathrm{d}$ and $\mathrm{f}$ electrons. Hence, we like to formulate as perspectives an extension to this work, especially in order to capture the impact of SIC onto the observed discrepancies.

\section{Perspectives: Self-interaction free functionals for atoms}

One of the fundamental assumptions of quantum chemistry is that an electron does not interact with itself. Applied to the density functional theory (DFT), this leads to a simple condition on the exact (and unknown) exchange-correlation functional: for any one-electron density distribution, the exchange-correlation (XC) energy must identically cancel the Coulomb self-interaction energy of the electron cloud.

Although this condition has been well-known since the very first steps in the development of DFT, satisfying it within model XC functionals has proven difficult. None of the approximate XC functionals, commonly used in quantum chemistry today, are self-interaction free. The presence of spurious self-interaction has been postulated as the reason behind some of the qualitative failures of approximate DFT.

Some time ago, Perdew and Zunger (PZ) proposed a simple correction, which removes the selfinteraction from a given approximate $\mathrm{XC}$ functional. Unfortunately, the $\mathrm{PZ}$ self-interaction correction (SIC) is not invariant to unitary transformations between the occupied molecular orbitals. This, in turn, leads to difficulties in practical implementation of the scheme, so that relatively few applications of PZ SIC to molecular systems have been reported.

\section{Self-interaction energy in DFT}

In Kohn-Sham DFT, the total electronic energy of the system is given by a sum of the kinetic energy, classical Coulomb energy of the electron charge distribution, and the exchange-correlation energy:

$$
\mathrm{E}_{\mathrm{tot}}^{\mathrm{KS}}=\sum_{\sigma=\alpha, \beta} \sum_{i=1}^{N_{\sigma}} n_{\sigma i}\left\langle\varphi_{\sigma i}\left|-\frac{1}{2} \hat{\Delta}\right| \varphi_{\sigma i}\right\rangle+\frac{1}{2} \int \frac{\rho\left(\vec{r}_{1}\right) \rho\left(\vec{r}_{2}\right)}{\vec{r}_{12}} d \vec{r}_{1} d \vec{r}_{2}+\int \mathrm{v}_{e x t}(\vec{r}) \rho(\vec{r}) d \vec{r}+E_{x c}\left[\rho_{\alpha}, \rho_{\beta}\right]
$$

At the same time, for a one-electron system, the to tal electronic energy is simply: 
$\mathrm{E}_{\mathrm{tot}}^{\mathrm{KS}}\left(1_{-}\right.$electron $)=\left\langle\varphi_{\mathrm{i} \sigma}\left|-\frac{1}{2} \hat{\Delta}\right| \varphi_{\mathrm{i} \sigma}\right\rangle+\int \mathrm{v}_{\text {ext }}(\vec{r}) \rho(\vec{r}) d \vec{r}$

Therefore, for any one-electron density $\rho$, the exact exchange-correlation functional must satisfy the following condition:

$$
\frac{1}{2} \int \frac{\rho\left(\vec{r}_{1}\right) \rho\left(\vec{r}_{2}\right)}{\vec{r}_{12}} d \vec{r}_{1} d \vec{r}_{2}+E_{x c}[\rho, 0]=0
$$

This condition is NOT satisfied by any popular approximate exchange-correlation functional

\section{Perdew-Zunger self-interaction correction}

$$
\mathrm{E}_{\mathrm{tot}}^{\mathrm{PZ}}=\mathrm{E}_{\mathrm{tot}}^{\mathrm{KS}}-\sum_{\sigma=\alpha, \beta} \sum_{i=1}^{N_{\sigma}}\left\{\frac{1}{2} \int \frac{\rho_{\sigma i}\left(\vec{r}_{1}\right) \rho_{\sigma i}\left(\vec{r}_{2}\right)}{\vec{r}_{12}} d \vec{r}_{1} d \vec{r}_{2}+E_{x c}\left[\rho_{\sigma i}, 0\right]\right\}
$$

The PZ correction has some desirable properties, most importantly:

- Correction (term is parentheses) vanishes for the exact functional $\mathrm{E}_{\mathrm{xc}}$

- The functional $\mathrm{E}^{\mathrm{PZ}}$ is exact for any one-electron system

- The XC potential has correct asymptotic behaviour at large $r$

At the same time,

- Total energy is orbital-dependent

- Exchange-correlation potentials are per-orbital

\section{Self-consistent implementation of PZ-SIC}

The non-trivial orbital dependence of the PZ-SIC energy leads to complications in practical selfconsistent implementation of the correction. Compare the outcomes of the standard variational minimization of $\mathrm{E}^{\mathrm{KS}}$ and $\mathrm{E}^{\mathrm{PZ}}$ :

Kohn-Sham: E Kot $_{\text {KS }}$

$$
\begin{aligned}
& \hat{\mathrm{f}}_{\sigma}^{\mathrm{KS}} \varphi_{\sigma i}=\varepsilon_{\sigma i} \varphi_{\sigma i} \\
& \hat{\mathrm{f}}_{\sigma}^{\mathrm{KS}}=-\frac{1}{2} \hat{\Delta}+\mathrm{v}_{\mathrm{c}}(\vec{r})+\mathrm{v}_{\mathrm{ext}}(\vec{r})+\mathrm{v}_{\mathrm{xc}, \sigma}(\vec{r})
\end{aligned}
$$

Perdew-Zunger: $E_{\text {tot }}^{\text {PZ }}$

$$
\begin{aligned}
& \hat{\mathrm{f}}_{\sigma i}^{\mathrm{PZ}} \varphi_{\sigma i}=\varepsilon_{\sigma i} \varphi_{\sigma i} \\
& \hat{\mathrm{f}}_{\sigma i}^{\mathrm{PZ}}=\hat{\mathrm{f}}_{\sigma i}^{\mathrm{KS}}+v_{\sigma i}^{P Z}(\vec{r})
\end{aligned}
$$




$$
v_{\sigma i}^{P Z}(\vec{r})=-\frac{\delta \mathrm{E}_{\mathrm{xc}}\left[\rho_{\sigma i}, 0\right]}{\delta \rho_{\sigma i}}-\int \frac{\rho_{\sigma i}\left(\vec{r}_{I}\right)}{\left|\vec{r}-\vec{r}_{I}\right|} \mathrm{d} \vec{r}_{I}
$$

The orbital dependence of the $\mathrm{f}^{\mathrm{PZ}}$ operator makes self-consistent implementation of PZ-SIC difficult, compared to Kohn-Sham DFT. However, the PZ self-interaction correction can also be implemented within an optimized effective potential (OEP) scheme, with eigen-equations formally identical to KS DFT:

$$
\begin{aligned}
\mathrm{E}_{\mathrm{tot}}^{\mathrm{PZ}} \Rightarrow \quad & \hat{\mathrm{f}}_{\sigma}^{\mathrm{OEP}} \varphi_{\sigma i}=\varepsilon_{\sigma i} \varphi_{\sigma i} \\
& \hat{\mathrm{f}}_{\sigma i}^{\mathrm{OEP}}=\hat{\mathrm{f}}_{\sigma i}^{\mathrm{KS}}+v_{\sigma}^{O E P}(\vec{r})
\end{aligned}
$$

where $v_{\sigma}^{O E P}$ is chosen to minimize $\mathrm{E}^{\mathrm{PZ}}$. Determining the exact OEP is difficult, and involves solving an integral equation on $\mathrm{v}^{\mathrm{OEP}}(\mathrm{r})$ :

$$
\sum_{i} n_{\sigma i} \int\left[v_{\sigma}^{O E P}\left(\vec{r}^{\prime}\right)-v_{\sigma i}^{P Z}\left(\vec{r}^{\prime}\right)\right] \sum_{j \neq i} \frac{\varphi_{j \sigma}\left(\vec{r}^{\prime}\right) \varphi_{j \sigma}(\vec{r})}{\varepsilon_{j \sigma^{-}} \varepsilon_{i \sigma}} d \vec{r}^{\prime}=0
$$

An exact solution of the OEP equation is only possible for small, and highly symmetric systems, such as atoms. Thus, the development of exact atomic OEP is certainly an interesting challenge in consideration of the previous discussion.

\begin{tabular}{|c|c|c|c|c|}
\hline Ion and method & $B / \mathrm{cm}^{-1}$ & $C / \mathrm{cm}^{-1}$ & $F_{2} / \mathrm{cm}^{-1}$ & $F_{4} / \mathrm{cm}^{-1}$ \\
\hline $\mathrm{Ti}^{L^{+}\lfloor j 0\rfloor}$ & 718 & 2629 & 1093.6 & 375.6 \\
\hline LDA & 870 & 3201 & 1327.3 & 457.3 \\
\hline PW91 & 870 & 3196 & 1326.6 & 456.6 \\
\hline PW91sd & 749 & 2729 & 1138.9 & 389.9 \\
\hline $\mathrm{V}^{2+}\lfloor 36]$ & 766 & 2855 & 1173.9 & 407.9 \\
\hline LDA & 944 & 3467 & 1439.3 & 495.3 \\
\hline
\end{tabular}

Table 1. Experimental and calculated Slater in tegrals $F_{k}$ for di-and trivalent d metal ions. The equivalent Racah parameters $B$ and $C$ are shown to facilitate the comparison with the spectroscopic literature. The sd suffix in the method indicates a calculation based on the fit of single determinant energies. 


\begin{tabular}{|c|c|c|c|c|}
\hline Ion and method & $B / \mathrm{cm}^{-1}$ & $C / \mathrm{cm}^{-1}$ & $F_{2} / \mathrm{cm}^{-1}$ & $F_{4} / \mathrm{cm}^{-1}$ \\
\hline PW91 & 944 & 3470 & 1439.7 & 495.7 \\
\hline PW91sd & 815 & 2961 & 1238.0 & 423.0 \\
\hline $\mathrm{V}^{3+}[30]$ & 861 & 4165 & 1456.0 & 595.0 \\
\hline LDA & 1074 & 4006 & 1646.3 & 572.3 \\
\hline PW91 & 1071 & 3994 & 1641.6 & 570.6 \\
\hline PW91sd & 918 & 3406 & 1404.6 & 486.6 \\
\hline $\mathrm{Cr}^{2+}[30]$ & 830 & 3430 & 1320.0 & 490.0 \\
\hline LDA & 1015 & 3727 & 1547.4 & 532.4 \\
\hline PW91 & 1016 & 3734 & 1549.4 & 533.4 \\
\hline PW91sd & 879 & 3189 & 1334.6 & 455.6 \\
\hline $\mathrm{Cr}^{3+}[\mathrm{s0}]$ & 1030 & 3850 & 1580.0 & 550.0 \\
\hline LDA & 1141 & 4246 & 1747.6 & 606.6 \\
\hline PW91 & 1140 & 4244 & 1746.3 & 606.3 \\
\hline PW91sd & 981 & 3610 & 1496.7 & 515.7 \\
\hline $\mathrm{Mn}^{L+}[30]$ & 960 & 3325 & 1435.0 & 475.0 \\
\hline LDA & 1085 & 3983 & 1654.0 & 569.0 \\
\hline PW91 & 1086 & 3991 & 1656.1 & 570.1 \\
\hline PW91sd & 942 & 3414 & 1429.7 & 487.7 \\
\hline $\mathrm{Mn}^{5+}[30]$ & 1140 & 3675 & 1665.0 & 525.0 \\
\hline LDA & 1207 & 4488 & 1848.1 & 641.1 \\
\hline PW91 & 1207 & 4490 & 1848.4 & 641.4 \\
\hline PW91sd & 1042 & 3841 & 1590.7 & 548.7 \\
\hline $\mathrm{Fe}^{2+}[50]$ & 1058 & 3901 & 1615.3 & 557.3 \\
\hline LDA & 1154 & 4233 & 1758.7 & 604.7 \\
\hline PW91 & 1155 & 4242 & 1761.0 & 606.0 \\
\hline PW91sd & 1003 & 3634 & 1522.1 & 519.1 \\
\hline
\end{tabular}




\begin{tabular}{|c|c|c|c|c|}
\hline Ion and method & $B / \mathrm{cm}^{-1}$ & $C / \mathrm{cm}^{-1}$ & $F_{2} / \mathrm{cm}^{-1}$ & $F_{4} / \mathrm{cm}^{-1}$ \\
\hline $\mathrm{Fe}^{5+\lfloor 21\rfloor}$ & 1015 & 4800 & 1700.7 & 685.7 \\
\hline LDA & 1273 & 4726 & 1948.1 & 675.1 \\
\hline PW91 & 1274 & 4731 & 1949.9 & 675.9 \\
\hline PW91sd & 1102 & 4054 & 1681.1 & 579.1 \\
\hline $\mathrm{Co}^{2+}\lfloor s 0]$ & 1115 & 4336 & 1734.4 & 619.4 \\
\hline LDA & 1222 & 4480 & 1862.0 & 640.0 \\
\hline PW91 & 1223 & 4489 & 1864.3 & 641.3 \\
\hline PW91sd & 1062 & 3850 & 1612.0 & 550.0 \\
\hline $\mathrm{Co}^{5+\lfloor 3 /\rfloor}$ & 1100 & 5120 & 1831.4 & 731.4 \\
\hline LDA & 1339 & 4963 & 2048.0 & 709.0 \\
\hline PW91 & 1340 & 4970 & 2050.0 & 710.0 \\
\hline PW91sd & 1161 & 4263 & 1770.0 & 609.0 \\
\hline $\mathrm{Ni}^{2+}\lfloor 30\rfloor$ & 1084 & 4831 & 1774.1 & 690.1 \\
\hline LDA & 1288 & 4721 & 1962.4 & 674.4 \\
\hline PW91 & 1289 & 4730 & 1964.7 & 675.7 \\
\hline PW91sd & 1120 & 4056 & 1699.4 & 579.4 \\
\hline $\mathrm{Ni}^{3+}$ & 1115 & 5450 & 1893.6 & 778.6 \\
\hline LDA & 1405 & 5206 & 2148.7 & 743.7 \\
\hline PW91 & 1406 & 5210 & 2150.3 & 744.3 \\
\hline PW91sd & 1219 & 4470 & 1857.6 & 638.6 \\
\hline \multicolumn{5}{|l|}{$\mathrm{Cu}^{3+}$} \\
\hline LDA & 1469 & 5438 & 2245.9 & 776.9 \\
\hline PW91 & 1470 & 5443 & 2247.6 & 777.6 \\
\hline PW91sd & 1275 & 4669 & 1942.0 & 667.0 \\
\hline \multicolumn{5}{|l|}{$\mathrm{Zr}^{2^{+}}$} \\
\hline LDA & 645 & 2542 & 1008.1 & 363.1 \\
\hline
\end{tabular}




\begin{tabular}{|c|c|c|c|c|}
\hline Ion and method & $\mathrm{B} / \mathrm{cm}^{-1}$ & $C / \mathrm{cm}^{-1}$ & $F_{2} / \mathrm{cm}^{-1}$ & $F_{4} / \mathrm{cm}^{-1}$ \\
\hline PW91 & 645 & 2542 & 1008.1 & 363.1 \\
\hline PW91sd & 553 & 2176 & 863.9 & 310.9 \\
\hline \multicolumn{5}{|l|}{$\mathrm{Nb}^{L^{+}}$} \\
\hline LDA & 697 & 2754 & 1090.4 & 393.4 \\
\hline PW91 & 699 & 2760 & 1093.3 & 394.3 \\
\hline PW91sd & 596 & 2357 & 932.7 & 336.7 \\
\hline $\mathrm{Nb}^{3+}\lfloor 36]$ & 602 & 1367 & 797.3 & 195.3 \\
\hline LDA & 761 & 3052 & 1197.0 & 436.0 \\
\hline PW91 & 761 & 3051 & 1196.9 & 435.9 \\
\hline PW91sd & 646 & 2633 & 1022.1 & 376.1 \\
\hline \multicolumn{5}{|l|}{$\mathrm{Mo}^{2+}$} \\
\hline LDA & 747 & 2953 & 1168.9 & 421.9 \\
\hline PW91 & 749 & 2966 & 1172.7 & 423.7 \\
\hline PW91sd & 638 & 2528 & 999.1 & 361.1 \\
\hline $\mathrm{Mo}^{5+}\lfloor$ ग/] & 610 & & & \\
\hline LDA & 809 & 3247 & 1272.9 & 463.9 \\
\hline PW91 & 810 & 3251 & 1274.4 & 464.4 \\
\hline PW91sd & 686 & 2798 & 1085.7 & 399.7 \\
\hline \multicolumn{5}{|l|}{$\mathrm{Tc}^{2+}$} \\
\hline LDA & 796 & 3152 & 1246.3 & 450.3 \\
\hline PW91 & 799 & 3165 & 1251.1 & 452.1 \\
\hline PW91sd & 678 & 2695 & 1063.0 & 385.0 \\
\hline \multicolumn{5}{|l|}{$\mathrm{Tc}^{3+}$} \\
\hline LDA & 855 & 3432 & 1345.3 & 490.3 \\
\hline PW91 & 857 & 3441 & 1348.6 & 491.6 \\
\hline PW91sd & 725 & 2951 & 1146.6 & 421.6 \\
\hline
\end{tabular}




\begin{tabular}{|c|c|c|c|c|}
\hline Ion and method & $B / \mathrm{cm}^{-1}$ & $\mathrm{C} / \mathrm{cm}^{-1}$ & $F_{2} / \mathrm{cm}^{-1}$ & $F_{4} / \mathrm{cm}^{-1}$ \\
\hline \multicolumn{5}{|l|}{$\mathrm{Ru}^{L^{+}}$} \\
\hline LDA & 843 & 3343 & 1320.6 & 477.6 \\
\hline PW91 & 846 & 3357 & 1325.6 & 479.6 \\
\hline PW91sd & 717 & 2856 & 1125.0 & 408.0 \\
\hline $\mathrm{Ru}^{3+}$ & & & 0.0 & 0.0 \\
\hline LDA & 900 & 3613 & 1416.1 & 516.1 \\
\hline PW91 & 903 & 3625 & 1420.9 & 517.9 \\
\hline PW91sd & 763 & 3100 & 1205.9 & 442.9 \\
\hline $\mathrm{Rh}^{L+}[3 /]$ & 620 & 4002 & 1191.7 & 571.7 \\
\hline LDA & 880 & 3529 & 1384.1 & 504.1 \\
\hline PW91 & 893 & 3545 & 1399.4 & 506.4 \\
\hline PW91sd & 755 & 3009 & 1184.9 & 429.9 \\
\hline $\mathrm{Rh}^{3+}\lfloor 5 /\rfloor$ & 720 & & & \\
\hline LDA & 945 & 3794 & 1487.0 & 542.0 \\
\hline PW91 & 948 & 3807 & 1491.9 & 543.9 \\
\hline PW91sd & 800 & 3247 & 1263.9 & 463.9 \\
\hline $\mathrm{Pd}^{2+}\lfloor 3 /\rfloor$ & 683 & 2620 & 1057.3 & 374.3 \\
\hline LDA & 935 & 3713 & 1465.4 & 530.4 \\
\hline PW91 & 938 & 3729 & 1470.7 & 532.7 \\
\hline PW91sd & 793 & 3154 & 1243.6 & 450.6 \\
\hline \multicolumn{5}{|l|}{$\mathrm{Pd}^{3+}$} \\
\hline LDA & 989 & 3971 & 1556.3 & 567.3 \\
\hline PW91 & 992 & 3984 & 1561.1 & 569.1 \\
\hline PW91sd & 836 & 3384 & 1319.4 & 483.4 \\
\hline \multicolumn{5}{|l|}{$\mathrm{Ag}^{5+}$} \\
\hline LDA & 1033 & 4146 & 1625.3 & 592.3 \\
\hline
\end{tabular}




\begin{tabular}{|c|c|c|c|c|}
\hline Ion and method & $B / \mathrm{cm}^{-1}$ & $C / \mathrm{cm}^{-1}$ & $F_{2} / \mathrm{cm}^{-1}$ & $F_{4} / \mathrm{cm}^{-1}$ \\
\hline PW91 & 1036 & 4162 & 1630.6 & 594.6 \\
\hline PW91sd & 872 & 3521 & 1375.0 & 503.0 \\
\hline \multicolumn{5}{|l|}{$\mathrm{Hf}^{\mathrm{L}^{+}}$} \\
\hline LDA & 628 & 2495 & 984.4 & 356.4 \\
\hline PW91 & 625 & 2482 & 979.6 & 354.6 \\
\hline PW91sd & 573 & 2274 & 897.9 & 324.9 \\
\hline \multicolumn{5}{|l|}{$\mathrm{Ta}^{2+}$} \\
\hline LDA & 668 & 2667 & 1049.0 & 381.0 \\
\hline PW91 & 667 & 2662 & 1047.3 & 380.3 \\
\hline PW91sd & 606 & 2425 & 952.4 & 346.4 \\
\hline \multicolumn{5}{|l|}{$\mathrm{Ta}^{3+}$} \\
\hline LDA & 728 & 2953 & 1149.9 & 421.9 \\
\hline PW91 & 726 & 2945 & 1146.7 & 420.7 \\
\hline PW91sd & 615 & 2493 & 971.1 & 356.1 \\
\hline \multicolumn{5}{|l|}{$\mathrm{W}^{L^{+}}$} \\
\hline LDA & 707 & 2829 & 1111.1 & 404.1 \\
\hline PW91 & 707 & 2831 & 1111.4 & 404.4 \\
\hline PW91sd & 600 & 2367 & 938.1 & 338.1 \\
\hline \multicolumn{5}{|l|}{$\mathrm{W}^{3+}$} \\
\hline LDA & 763 & 3101 & 1206.0 & 443.0 \\
\hline PW91 & 762 & 3098 & 1204.6 & 442.6 \\
\hline PW91sd & 643 & 2618 & 1017.0 & 374.0 \\
\hline \multicolumn{5}{|l|}{$\operatorname{Re}^{2+}$} \\
\hline LDA & 744 & 2989 & 1171.0 & 427.0 \\
\hline PW91 & 745 & 2993 & 1172.6 & 427.6 \\
\hline PW91sd & 630 & 2508 & 988.3 & 358.3 \\
\hline
\end{tabular}




\begin{tabular}{|c|c|c|c|c|}
\hline Ion and method & $B / \mathrm{cm}^{-1}$ & $C / \mathrm{cm}^{-1}$ & $F_{2} / \mathrm{cm}^{-1}$ & $F_{4} / \mathrm{cm}^{-1}$ \\
\hline \multicolumn{5}{|l|}{$\operatorname{Re}^{5+}$} \\
\hline LDA & 797 & 3245 & 1260.6 & 463.6 \\
\hline PW91 & 797 & 3247 & 1260.9 & 463.9 \\
\hline PW91sd & 671 & 2739 & 1062.3 & 391.3 \\
\hline \multicolumn{5}{|l|}{$\mathrm{Os}^{2+}$} \\
\hline LDA & 780 & 3144 & 1229.1 & 449.1 \\
\hline PW91 & 782 & 3151 & 1232.1 & 450.1 \\
\hline PW91sd & 660 & 2641 & 1037.3 & 377.3 \\
\hline \multicolumn{5}{|l|}{$\mathrm{Os}^{3+}$} \\
\hline LDA & 830 & 3387 & 1313.9 & 483.9 \\
\hline PW91 & 831 & 3392 & 1315.6 & 484.6 \\
\hline PW91sd & 698 & 2857 & 1106.1 & 408.1 \\
\hline \multicolumn{5}{|l|}{$\operatorname{Ir}^{2+}$} \\
\hline LDA & 816 & 3296 & 1286.9 & 470.9 \\
\hline PW91 & 817 & 3303 & 1288.9 & 471.9 \\
\hline PW91sd & 688 & 2768 & 1083.4 & 395.4 \\
\hline $\operatorname{Ir}^{3+}\lfloor 3 /\rfloor$ & 660 & & & \\
\hline LDA & 863 & 3528 & 1367.0 & 504.0 \\
\hline PW91 & 864 & 3535 & 1369.0 & 505.0 \\
\hline PW91sd & 725 & 2972 & 1149.6 & 424.6 \\
\hline $\mathrm{Pt}^{L+}\lfloor 3 /\rfloor$ & 600 & & & \\
\hline LDA & 850 & 3442 & 1341.7 & 491.7 \\
\hline PW91 & 852 & 3451 & 1345.0 & 493.0 \\
\hline PW91sd & 772 & 2885 & 1184.1 & 412.1 \\
\hline \multicolumn{5}{|l|}{$\mathrm{Pt}^{3+}$} \\
\hline LDA & 896 & 3666 & 1419.7 & 523.7 \\
\hline
\end{tabular}




\begin{tabular}{ccccc}
\hline Ion and method & $\boldsymbol{B} / \mathbf{c m}^{-\boldsymbol{1}}$ & $\boldsymbol{C} / \mathbf{c m}^{-\mathbf{1}}$ & $\boldsymbol{F}_{2} / \mathbf{c m}^{-\mathbf{1}}$ & $\boldsymbol{F}_{4} / \mathbf{c m}^{-\boldsymbol{I}}$ \\
\hline PW91 & 897 & 3674 & 1421.9 & 524.9 \\
PW91sd & 752 & 3083 & 1192.4 & 440.4 \\
$\mathrm{Au}^{\text {5+ }}$ & & & & \\
LDA & 928 & 3802 & 1471.1 & 543.1 \\
PW91 & 929 & 3811 & 1473.4 & 544.4 \\
PW91sd & 778 & 3187 & 1233.3 & 455.3 \\
\hline
\end{tabular}

Table 2. Experimental and calculated Slater integrals $F_{k}$ for lanthanide(III) and actinide(III) ions. The Dirac-Fock results are taken from the work of Freeman. ${ }^{[27]}$ The sd suffix in the method indicates a calculation based on the fit of single determinant energies.

\begin{tabular}{|c|c|c|c|c|}
\hline Ion and method & $F_{0} / \mathrm{cm}^{-1}$ & $F_{2} / \mathrm{cm}^{-1}$ & $F_{4} / \mathrm{cm}^{-1}$ & $F_{6} / \mathrm{cm}^{-1}$ \\
\hline $\operatorname{Pr}^{3+}$ & N/A & 322 & 49.7 & 4.9 \\
\hline $\operatorname{Pr}^{3+}(a q)^{\lfloor<\angle\rfloor}$ & N/A & 305.2 & 46.3 & 4.4 \\
\hline $\mathrm{X} \alpha$ & 20257 & 421.4 & 54.3 & 5.77 \\
\hline VWN & 20183 & 419.7 & 54.1 & 5.75 \\
\hline \multicolumn{5}{|l|}{ VWNsd (with } \\
\hline fro zen core) & N/A & 367.6 & 35.4 & 1.55 \\
\hline VWNsd & N/A & 374.9 & 36 & 1.57 \\
\hline BP86 & 20155 & 419.1 & 54 & 5.74 \\
\hline PW91 & 20153 & 419 & 54 & 5.74 \\
\hline \multicolumn{5}{|l|}{ PW91sd (with } \\
\hline frozen core) & N/A & 399.7 & 40.3 & 0.73 \\
\hline PW91sd & $\mathrm{N} / \mathrm{A}$ & 385.9 & 38.4 & 0.6 \\
\hline LB94 & 20876 & 437.6 & 56.6 & 6.02 \\
\hline $\mathrm{Nd}^{J+}(\mathrm{aq})^{\lfloor\ll\rfloor}$ & N/A & 321.8 & 46.3 & 4.7 \\
\hline $\mathrm{X} \alpha$ & 21100 & 439.6 & 56.7 & 6.02 \\
\hline VWN & 21025 & 437.8 & 56.5 & 6 \\
\hline
\end{tabular}




\begin{tabular}{|c|c|c|c|c|}
\hline Ion and method & $F_{0} / \mathrm{cm}^{-1}$ & $F_{2} / \mathrm{cm}^{-1}$ & $F_{4} / \mathrm{cm}^{-1}$ & $F_{6} / \mathrm{cm}^{-1}$ \\
\hline VWNsd & N/A & 391 & 37.3 & 1.62 \\
\hline PW91 & 21000 & 437.2 & 56.4 & 5.99 \\
\hline PW91sd & N/A & 402.9 & 40 & 0.64 \\
\hline LB94 & 21653 & 454.1 & 58.7 & 6.24 \\
\hline Dirac-Fock & 21497 & 454.4 & 58.9 & 6.3 \\
\hline $\mathrm{Pm}^{3+}(\mathrm{aq})^{\lfloor\ll\rfloor}$ & N/A & 338.3 & 49.8 & 4.5 \\
\hline $\mathrm{X} \alpha$ & 21897 & 456.6 & 58.9 & 6.26 \\
\hline VWN & 21821 & 454.8 & 58.7 & 6.23 \\
\hline VWNsd & N/A & 406.1 & 38.5 & 1.68 \\
\hline PW91 & 21798 & 454.3 & 58.6 & 6.23 \\
\hline PW91sd & N/A & 418.8 & 41.5 & 0.68 \\
\hline LB94 & 22396 & 469.8 & 60.8 & 6.46 \\
\hline $\mathrm{Sm}^{J+}(\mathrm{aq})^{\lfloor<\rfloor}$ & N/A & 364.5 & 56.7 & 5.4 \\
\hline $\mathrm{X} \alpha$ & 22660 & 472.8 & 61 & 6.49 \\
\hline VWN & 22583 & 470.9 & 60.8 & 6.46 \\
\hline VWNsd & N/A & 420.5 & 39.7 & 1.72 \\
\hline PW91 & 22565 & 470.6 & 60.7 & 6.45 \\
\hline PW91sd & N/A & 433.9 & 42.9 & 0.71 \\
\hline LB94 & 23114 & 484.8 & 62.7 & 6.67 \\
\hline Dirac-Fock $^{[27]}$ & 23027 & 487 & 63.1 & 6.7 \\
\hline $\mathrm{Eu}^{3+}(\mathrm{aq})^{\lfloor<3\rfloor}$ & N/A & 369.6 & 56.2 & 5.6 \\
\hline $\mathrm{X} \alpha$ & 23408 & 488.6 & 63.1 & 6.7 \\
\hline VWN & 23331 & 486.6 & 62.8 & 6.67 \\
\hline VWNsd & N/A & 434.4 & 40.7 & 1.77 \\
\hline BP86 & 23314 & 486.4 & 62.8 & 6.67 \\
\hline PW91 & 23315 & 486.4 & 62.8 & 6.67 \\
\hline
\end{tabular}




\begin{tabular}{|c|c|c|c|c|}
\hline Ion and method & $F_{0} / \mathrm{cm}^{-1}$ & $F_{2} / \mathrm{cm}^{-1}$ & $F_{4} / \mathrm{cm}^{-1}$ & $F_{6} / \mathrm{cm}^{-1}$ \\
\hline PW91sd & $\mathrm{N} / \mathrm{A}$ & 448.5 & 44.2 & 0.75 \\
\hline LB94 & 23824 & 499.6 & 64.6 & 6.87 \\
\hline Dirac-Fock $^{[27]}$ & 23754 & 502.3 & 65.1 & 6.9 \\
\hline $\mathrm{Gd}^{J+}(\mathrm{aq})^{\lfloor\nu\rfloor}$ & N/A & 385 & 57.7 & 5.8 \\
\hline $\mathrm{X} \alpha$ & 24124 & 503.6 & 65 & 6.91 \\
\hline VWN & 24045 & 501.6 & 64.8 & 6.88 \\
\hline VWNsd & N/A & 447.6 & 41.7 & 1.81 \\
\hline BP86 & 24030 & 501.4 & 64.7 & 6.88 \\
\hline PW91 & 24030 & 501.4 & 64.7 & 6.88 \\
\hline PW91sd & N/A & 462.4 & 45.4 & 0.78 \\
\hline LB94 & 24508 & 513.7 & 66.4 & 7.06 \\
\hline Dirac-Fock $^{[27]}$ & 24461 & 517.1 & 67 & 7.1 \\
\hline $\mathrm{Tb}^{J+}(\mathrm{aq})^{\lfloor<4\rfloor}$ & N/A & 401.6 & 60.8 & 6 \\
\hline $\mathrm{X} \alpha$ & 24828 & 518.3 & 66.9 & 7.11 \\
\hline VWN & 24748 & 516.3 & 66.6 & 7.09 \\
\hline VWNsd & $\mathrm{N} / \mathrm{A}$ & 460.6 & 42.6 & 1.84 \\
\hline PW91 & 24733 & 516.1 & 66.6 & 7.08 \\
\hline PW91sd & N/A & 475.8 & 46.7 & 0.81 \\
\hline LB94 & 25183 & 527.6 & 68.2 & 7.25 \\
\hline Dirac-Fock $^{[27]}$ & 25150 & 531.5 & 68.9 & 7.3 \\
\hline $\mathrm{Dy}^{3+}(\mathrm{aq})^{\lfloor\lfloor\rfloor}$ & $\mathrm{N} / \mathrm{A}$ & 407.7 & 60.5 & 6.3 \\
\hline $\mathrm{X} \alpha$ & 25514 & 532.5 & 58.8 & 7.31 \\
\hline VWN & 25434 & 530.5 & 68.5 & 7.27 \\
\hline VWNsd & N/A & 473.1 & 43.5 & 1.87 \\
\hline PW91 & 25421 & 530.3 & 68.5 & 7.27 \\
\hline PW91sd & N/A & 488.8 & 47.7 & 0.84 \\
\hline
\end{tabular}




\begin{tabular}{|c|c|c|c|c|}
\hline Ion and method & $F_{0} / \mathrm{cm}^{-1}$ & $F_{2} / \mathrm{cm}^{-1}$ & $F_{4} / \mathrm{cm}^{-1}$ & $F_{6} / \mathrm{cm}^{-1}$ \\
\hline LB94 & 25844 & 541.2 & 70 & 7.44 \\
\hline Dirac-Fock $^{[27]}$ & 25825 & 545.5 & 70.7 & 7.5 \\
\hline $\mathrm{Ho}^{3+}(\mathrm{aq})^{\lfloor\ll\rfloor}$ & N/A & 419.8 & 65.1 & 6.8 \\
\hline $\mathrm{X} \alpha$ & 26186 & 546.5 & 70.6 & 7.5 \\
\hline VWN & 26104 & 544.4 & 70.3 & 7.47 \\
\hline VWNsd & N/A & 485.3 & 44.4 & 1.9 \\
\hline PW91 & 26093 & 544.3 & 70.3 & 7.47 \\
\hline PW91sd & $\mathrm{N} / \mathrm{A}$ & 501.6 & 48.8 & 0.87 \\
\hline LB94 & 26493 & 554.6 & 71.7 & 7.62 \\
\hline Dirac-Fock $^{[27]}$ & 26484 & 559.1 & 72.5 & 7.7 \\
\hline $\mathrm{Er}^{3+}$ & N/A & 441.7 & 68.3 & 7.5 \\
\hline $\operatorname{Er}^{3+}(\mathrm{aq})^{\lfloor<2\rfloor}$ & N/A & 440.8 & 66.8 & 7.3 \\
\hline $\mathrm{X} \alpha$ & 26846 & 560.1 & 72.3 & 7.68 \\
\hline VWN & 26764 & 558 & 72 & 7.65 \\
\hline VWNsd & $\mathrm{N} / \mathrm{A}$ & 497.3 & 45.2 & 1.93 \\
\hline BP86 & 26755 & 558 & 72 & 7.65 \\
\hline PW91 & 26754 & 558 & 72 & 7.65 \\
\hline PW91sd & N/A & 514 & 49.8 & 0.89 \\
\hline LB94 & 27133 & 567.7 & 73.4 & 7.8 \\
\hline Dirac-Fock $^{[27]}$ & 27134 & 572.5 & 74.2 & 7.9 \\
\hline $\operatorname{Tm}^{3+}(\mathrm{aq})^{\lfloor\ll\rfloor}$ & $\mathrm{N} / \mathrm{A}$ & 461.7 & 70.7 & 7.8 \\
\hline $\mathrm{X} \alpha$ & 27499 & 573.5 & 74 & 7.87 \\
\hline VWN & 27416 & 571.4 & 73.8 & 7.84 \\
\hline VWNsd & N/A & 509 & 46 & 1.95 \\
\hline BP86 & 24709 & 571.4 & 73.8 & 7.84 \\
\hline PW91 & 27410 & 571.4 & 73.8 & 7.84 \\
\hline
\end{tabular}




\begin{tabular}{|c|c|c|c|c|}
\hline Ion and method & $F_{0} / \mathrm{cm}^{-1}$ & $F_{2} / \mathrm{cm}^{-1}$ & $F_{4} / \mathrm{cm}^{-1}$ & $F_{6} / \mathrm{cm}^{-1}$ \\
\hline PW91sd & $\mathrm{N} / \mathrm{A}$ & 526.1 & 50.8 & 0.91 \\
\hline LB94 & 27769 & 580.6 & 75 & 7.97 \\
\hline Dirac-Fock $^{[27]}$ & 27773 & 585.6 & 75.9 & 8.1 \\
\hline \multicolumn{5}{|l|}{$\mathrm{Pa}^{3+}$} \\
\hline VWN (4 zetas) & 180040 & 397.1 & 52.9 & 5.7 \\
\hline PW91 & 179870 & 396.6 & 52.8 & 5.69 \\
\hline $\mathrm{U}^{3+}$ & $\mathrm{N} / \mathrm{A}$ & 176 & 30.3 & 3.14 \\
\hline $\mathrm{U}^{3+}$ & $\mathrm{N} / \mathrm{A}$ & 196 & & \\
\hline VWN (4 zetas) & 187150 & 413.4 & 55.2 & 5.95 \\
\hline PW91 & 187020 & 413 & 55.1 & 5.95 \\
\hline $\mathrm{Np}^{5+}$ & N/A & 225 & & \\
\hline VWN (4 zetas) & 193800 & 428.3 & 57.2 & 6.18 \\
\hline PW91 & 193730 & 428.2 & 57.2 & 6.18 \\
\hline $\mathrm{Pu}^{3+}$ & N/A & 240 & & \\
\hline VWN (4 zetas) & 200110 & 442.6 & 59.2 & 6.4 \\
\hline PW91 & 200090 & 442.6 & 59.2 & 6.4 \\
\hline $\mathrm{Am}^{3+}$ & $\mathrm{N} / \mathrm{A}$ & 260 & & \\
\hline VWN (4 zetas) & 206160 & 456.1 & 61.1 & 6.61 \\
\hline PW91 & 206170 & 456.2 & 61.1 & 6.61 \\
\hline $\mathrm{Cm}^{3+}$ & N/A & 251 & & \\
\hline $\mathrm{Cm}^{3+}$ & N/A & 280 & & \\
\hline $\mathrm{Cm}^{3+}$ & N/A & 251.6 & 40.55 & 4.31 \\
\hline VWN (4 zetas) & 211980 & 469.1 & 62.9 & 6.8 \\
\hline PW91 & 212020 & 469.3 & 62.9 & 6.81 \\
\hline $\mathrm{Bk}^{3+}$ & N/A & 263.1 & 41.2 & 4.58 \\
\hline VWN (5 zetas) & 172480 & 379.5 & 51.1 & 5.54 \\
\hline
\end{tabular}




\begin{tabular}{|c|c|c|c|c|}
\hline Ion and method & $F_{0} / \mathrm{cm}^{-1}$ & $F_{2} / \mathrm{cm}^{-1}$ & $F_{4} / \mathrm{cm}^{-1}$ & $F_{6} / \mathrm{cm}^{-1}$ \\
\hline PW91 & 172530 & 379.7 & 51.1 & 5.55 \\
\hline \multicolumn{5}{|l|}{$\mathrm{Cf}^{3+}$} \\
\hline VWN (5 zetas) & 177960 & 391.6 & 52.8 & 5.73 \\
\hline PW91 & 178030 & 392 & 52.8 & 5.73 \\
\hline $\mathrm{Es}^{3+}$ & $\mathrm{N} / \mathrm{A}$ & 338 & & \\
\hline $\mathrm{Es}^{3+}$ & $\mathrm{N} / \mathrm{A}$ & 280 & & \\
\hline VWN (5 zetas) & 183290 & 403.5 & 54.4 & 5.91 \\
\hline PW91 & 183380 & 403.9 & 54.4 & 5.91 \\
\hline $\mathrm{Fm}^{3+}$ & N/A & 292.7 & 47.8 & 5.13 \\
\hline VWN (5 zetas) & 188510 & 415 & 56 & 6.08 \\
\hline PW91 & 188610 & 415.4 & 56 & 6.09 \\
\hline
\end{tabular}

\section{Acknowledgement:}

This work was supported by the Swiss National Science Foundation and by the State Secretariat for Education and Science (COST D26).

\section{Bibliography}

[1] M. Atanasov, C. A. Daul, C. Rauzy, Structure and Bonding 2004, 106, 97.

[2] M. Atanasov, C. A. Daul, C. Rauzy, Chem. Phys. Lett. 2003, 367, 737.

[3] M. Atanasov, C. Rauzy, P. Baettig, C. A. Daul, Int. J. Quant. Chem. 2005, 102, 119.

[4] M. Atanasov, C. A. Daul, H. U. Güdel, T. A. Wesolowski, M. Zbiri, Inorg, Chem. 2005, 44, 2954.

[5] A. Borel, L. Helm, C. A. Daul, Chem. Phys. Lett. 2004, 383, 584.

[6] P. Hohenberg, W. Kohn, Phys. Rev. 1964, 136, B864.

[7] W. Kohn, L. J. Sham, Phys. Rev. 1965, 140, A1133.

[8] J. C. Slater, Phys. Rev. 1951, 81, 385. 
[9] R. G. Parr, W. Yang, Density Functional Theory, Oxford University Press, New York, 1989.

[10] R. M. Dreizler, E. K. U. Gross, Density Functional Theory, Springer, Berlin, 1990.

[11] W. Koch, M. C. Holthau sen, A Chemist's Guide to Density Functional Theory, Wiley-VCH, New York, 2000.

[12] V. Weber, C. A. Daul, R. Baltensperger, Comp. Phys. Commun. 2004, 163, 133.

[13] E. J. Baerends, 2001 ed., Scientific Computing and Modelling, Amsterdam, 2001.

[14] S. H. Vosko, L. Wilk, M. Nusair, Can. J. Phys. 1980, 58, 1200.

[15] J. P. Perdew, J. A. Chevary, S. H. Vosko, K. A. Jackson, M. R. Pederson, D. J. Singh, C. Fiolhais, Phys. Rev. B 1992, 46, 6671.

[16] E. van Lenthe, P. E. S. Wormer, A. van der Avoird, J. Chem. Phys. 1997, 107, 2488.

[17] J. C. Slater, The Self-Consistent Field for Molecules and Solids, McGraw-Hill, New York, 1974.

[18] R. van Leuween, E. J. Baerends, Phys. Rev. A 1994, 49, 2421.

[19] A. D. Becke, J. Chem. Phys. 1988, 88, 1053.

[20] J. Perdew, Phys. Rev. B 1986, 33, 8822.

[21] I. B. Bersuker, in Electronic Structure and Properties of Transition Metal Compounds, Wiley \& Sons, New York, 1996, pp. 104.

[22] W. T. Carnall, P. R. Fields, K. Kajnak, J. Chem. Phys. 1968, 49, 4424.

[23] W. T. Carnall, P. R. Fields, J. Chem. Phys. 1968, 49, 4450.

[24] W. T. Carnall, P. R. Fields, K. Rajnak, J. Chem. Phys. 1968, 49, 4447.

[25] W. T. Carnall, P. R. Fields, K. Rajnak, J. Chem. Phys. 1968, 49, 4443.

[26] A. J. Freeman, R. E. Watson, Phys. Rev. 1962, 127, 2058.

[27] A. J. Freeman, J. P. Desclaux, J. Magnetism Magn. Mat. 1979, 12, 11.

[28] A. D. Becke, J. Chem. Phys. 1993, 98, 5648.

[29] C. E. Schäffer, C. K. Jørgensen, J. Inorg. Nucl. Chem. 1958, 8, 143.

[30] F. Neese, E. I. Solomon, Inorg. Chem. 1998, 37, 6568.

[31] C. Anthon, C. E. Schäffer, Coord. Chem. Rev. 2002, 226, 17.

[32] C. K. Jørgensen, R. Papallardo, H.-H. Schmidtke, J. Chem. Phys. 1963, 39, 1422.

[33] J. C. Barnes, J. Chem. Soc. A 1966, 667.

[34] J. P. Perdew, A. Zunger, J. Chem. Phys. 1981, 23, 23.

[35] S. Patchkovskii, J. Autschbach, T. Ziegler, J. Chem. Phys. 2001, 115, 26.

[36] J. S. Griffith, in The Theory of Transition Metal Ions, University Press, Cambridge, 1961, pp. 437.

[37] A. B. P. Lever, in Inorganic Electronic Spectroscopy, 2nd. ed., Elsevier, Amsterdam, 1984, p. 115 . 


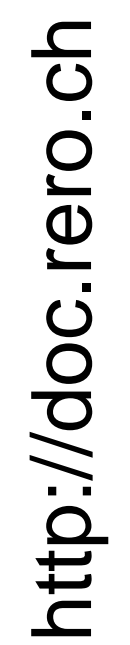


Appendix: Calculation of $\mathrm{W}_{\mathrm{k}}(\mathrm{a}, \mathrm{b}, \mathrm{c}, \mathrm{d})$ over Slater Type Orbitals

The scripts below are written for OCTAVE / MATLAB

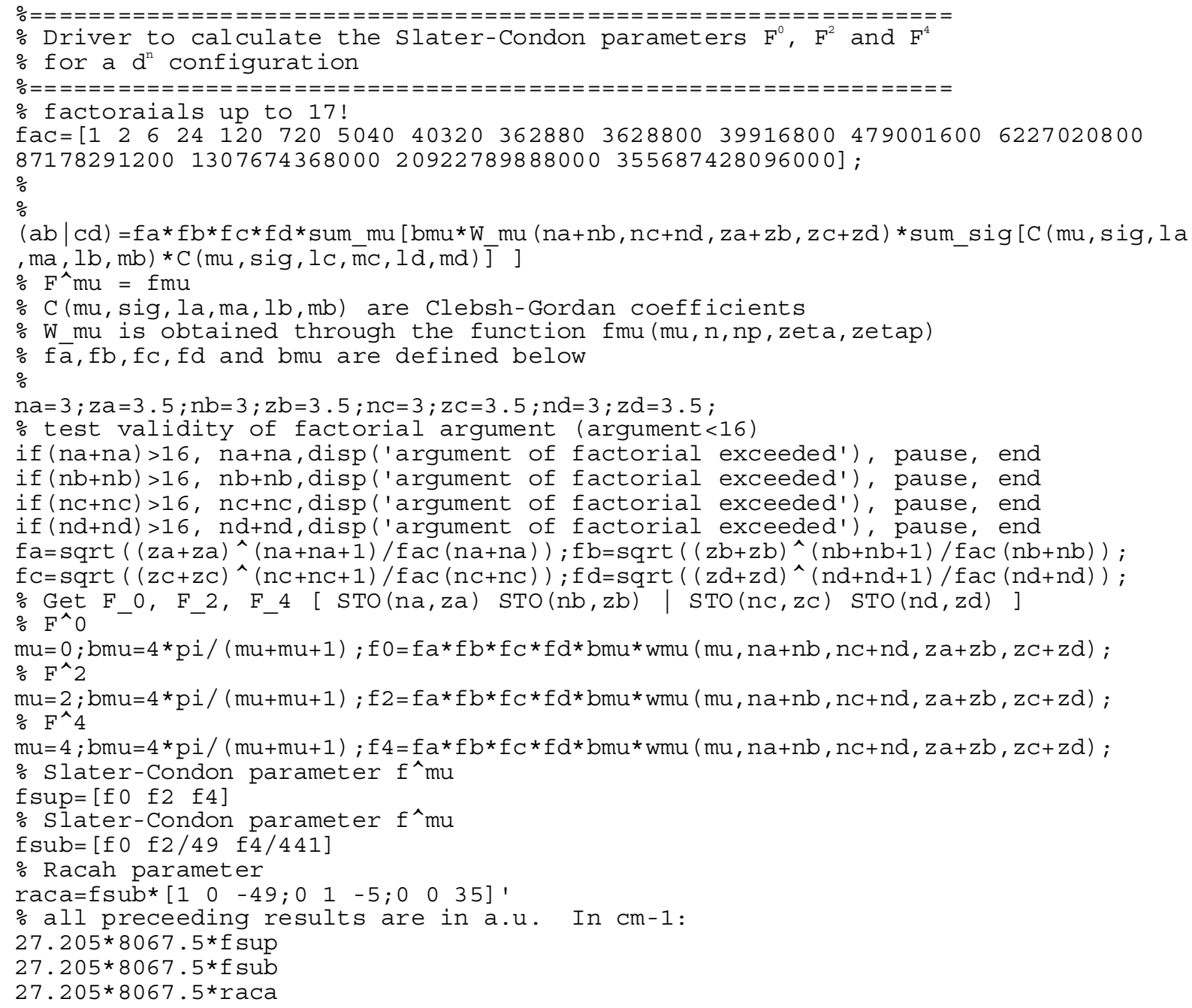


